\title{
Anti-Periodic Dynamics of Quaternion-Valued Fuzzy Cellular Neural Networks with Time-Varying Delays on Time Scales
}

\author{
Shiping Shen,, Bing $\mathrm{Li}^{1}{ }^{2}$ and Yongkun $\mathrm{Li}{ }^{1}{ }^{1}$ \\ ${ }^{1}$ Department of Mathematics, Yunnan University, Kunming, Yunnan 650091, China \\ ${ }^{2}$ School of Mathematics and Computer Science, Yunnan Nationalities University, Kunming, Yunnan 650500, China \\ Correspondence should be addressed to Yongkun Li; yklie@ynu.edu.cn
}

Received 20 March 2018; Revised 21 May 2018; Accepted 29 May 2018; Published 28 June 2018

Academic Editor: J. R. Torregrosa

Copyright (c) 2018 Shiping Shen et al. This is an open access article distributed under the Creative Commons Attribution License, which permits unrestricted use, distribution, and reproduction in any medium, provided the original work is properly cited.

A class of quaternion-valued fuzzy cellular neural networks with time-varying delays on time scales is proposed. Based on inequality analysis techniques on time scales, a fixed point theorem and the theory of calculus on time scales, the existence, and global exponential stability of anti-periodic solutions for this class of neural networks are established. The obtained results are completely new and supplement to the known results. Finally, a numerical example is given to illustrate the feasibility of our results.

\section{Introduction}

Since Yang and Yang [1] first introduced fuzzy cellular neural networks (FCNNs) combining fuzzy operations (fuzzy AND and fuzzy OR) with cellular neural networks, FCNNs have been successfully applied in many fields such as physics, chemistry, biology, economics, sociology, medicine, and meteorology [2]. Because all of their applications heavily rely on their dynamics, in recent years, a lot of meaningful results regarding the dynamics of them are obtained by many researchers (see [3-11] and reference therein). For instance, authors in [8] studied the global stability of equilibria of FCNNs, authors in [9] obtained some sufficient conditions for the existence and stability of a unique periodic solution of FCNNs, authors in [10] investigated the existence and global exponential stability of anti-periodic solutions for neutral type FCNNs with time-varying delays and $D$ operator on time scales, the author in [11] studied the almost periodicity of FCNNs with multi-proportional delays, and in other accounts authors investigated other behaviors of FCNNs with time delays.

On the one hand, because quaternion-valued neural networks (QVNNs) as an extension of the real-valued neural networks and complex-valued neural networks can be extensively applied to the fields of robotics, attitude control of satellites, computer graphics, ensemble control, color night vision, and image compression ([12-14]) and one of the benefits by using quaternion is the three-dimensional geometrical affine transformation that can be represented efficiently and compactly, the study of dynamical behaviors for QVNNs has received much attention of many scholars and some good results have been obtained for the stability [15-19], dissipativity [20], periodicity [21], pseduo almost periodicity [22], and synchronization of QVNNs [23, 24].

On the other hand, as we know, a special case of the quasi-periodicity of functions is the anti-periodicity and $\omega$-anti-periodic functions are $2 \omega$-periodic functions, but not all periodic functions are anti-periodic ones. Since the signal transmission process of neural networks can often be described as an anti-periodic process, the problem of antiperiodic solutions for various types of neural networks has been investigated by many authors ([10, 25-34]).

Moreover, continuous time and discrete time systems are very important in implementation and applications. In addition, in a realistic system, the interaction among agents can happen at any time, and maybe some continuous time intervals accompany some discrete moments. So it is necessary and significant to consider both continuous time and discrete time cases at the same time in networked systems. Fortunately, the time scale theory, which was introduced by Hilger [35], can unify the study of continuous and discrete analysis, and the study of dynamic equations on time scales 
can contain, link, and extend the classical theory of differential and difference equations [36]. Recently, the theory of time scale calculus has been applied in real-valued neural networks [37-43] and complex-valued networks [44]. However, to the best of our knowledge, the existence and global stability of anti-periodic solutions of quaternion-valued fuzzy cellular neural networks (QVFCNNs) on time scales have not been considered yet. Besides, it is well known that time delays are unavoidable in real neural network systems and they may cause the changes of the dynamical behaviors of neural networks [11, 25, 40, 41].

Motivated by the above discussion, our main aim of this paper is to study the existence and global stability of antiperiodic solutions of QVFCNNs with time-varying delays. The innovation points of this paper are summarized as follows:

(1) We propose a class of QVFCNNs with time-varying delays on time scales which can unify the continuous time and discrete time cases of QVFCNNs and, what is more, which can contain the QVFCNNs that their time argument may vary in some continuous time intervals accompanying some discrete moments.

(2) The QVFCNNs proposed in this paper contain realvalued FCNNs and complex-valued FCNNs as their special cases.

(3) Our methods of establishing the existence of antiperiodic solutions of QVFCNNs with time-varying delays on time scales are different from those used in $[10,25-34]$ and can be used to study other types of neural networks.

(4) Our results are completely new and supplement to the known results, and our results show that if the coefficients of leakage terms of QVFCNNs with timevarying delays on time scales are positive regressive, then both the continuous time and discrete time QVFCNNs with time-varying delays have the same dynamics for the anti-periodicity.

This paper is organized as follows. In Section 2, we give the model description and introduce some definitions and preliminary lemmas and transform the quaternionvalued system (10) into an equivalent real-valued system. In Section 3, we establish the existence of anti-periodic solutions of the considered network based on a fixed point theorem. In Section 4, by using some inequality techniques, we derive some sufficient conditions for the global exponential stability of anti-periodic solutions of the considered network. In Section 5, we give an example to show the feasibility and effectiveness of our main results. This paper ends with a brief conclusion in Section 6.

\section{Model Description and Preliminaries}

The quaternion was invented in 1843 by Hamilton [45]. The skew field of quaternion is denoted by

$$
\mathbb{Q}:=\left\{q=q^{R}+q^{I} i+q^{J} j+q^{K} k\right\},
$$

where $q^{R}, q^{I}, q^{J}, q^{K}$ are real numbers and the elements $i, j$, and $k$ obey the Hamilton's multiplication rules:

$$
\begin{aligned}
& i j=-j i=k, \\
& j k=-k j=i, \\
& k i=-i k=j, \\
& i^{2}=j^{2}=k^{2}=i j k=-1 .
\end{aligned}
$$

A time scale $\mathbb{T}$ is an arbitrary nonempty closed subset of the real set $\mathbb{R}$ with the topology and ordering inherited from $\mathbb{R}$. The forward and the backward jump operators $\sigma, \rho: \mathbb{T} \longrightarrow$ $\mathbb{T}$ are defined by

$$
\begin{aligned}
& \sigma(t)=\inf \{s \in \mathbb{T}, s>t\}, \\
& \rho(t)=\sup \{s \in \mathbb{T}, s<t\},
\end{aligned}
$$

$\forall t \in \mathbb{T}$.

The graininess function $\mu: \mathbb{T} \longrightarrow[0, \infty)$ is defined by $\mu(t)=\sigma(t)-t$. The point $t \in \mathbb{\mathbb { T }}$ is called left-dense, leftscattered, right-dense, or right-scattered if $\rho(t)=t, \rho(t)<t$, $\sigma(t)=t$ or $\sigma(t)>t$, respectively. Points that are right-dense and left-dense at the same time are called dense. If $\mathbb{T}$ has a left-scattered maximum $m$, define $\mathbb{T}^{\kappa}=\mathbb{T}-\{m\}$; otherwise, set $\mathbb{T}^{\kappa}=\mathbb{T}$. If $\mathbb{T}$ has a right-scattered maximum $m$, define $\mathbb{T}_{\kappa}=\mathbb{T}-\{m\}$; otherwise, set $\mathbb{T}_{\kappa}=\mathbb{T}$.

Assume that $f: \mathbb{T} \longrightarrow \mathbb{R}$ is a function and let $t \in \mathbb{T}^{k}$. Then we define $f^{\Delta}(t)$ to be the number (provided it exists) with the property that given any $\varepsilon>0$, there is a neighborhood $\mathrm{U}$ of $\mathrm{t}$ (i.e., $U=(t-\delta, t+\delta) \cap \mathbb{T}$ for some $\delta>0)$ such that

$$
\left|f(\sigma(t))-f(s)-f^{\Delta}(t)(\sigma(t)-s)\right| \leq \varepsilon|\sigma(t)-s|
$$

for all $s \in U$. We call $f^{\Delta}(t)$ the delta derivative of $f$ at $t$.

Lemma 1 (see [36]). Let $a \in \mathbb{T}^{k}, b \in \mathbb{T}$ and assume that $f$ : $\mathbb{T} \times \mathbb{T}^{k} \longrightarrow \mathbb{R}$ is continuous at $(t, t)$, where $t \in \mathbb{T}^{k}$ with $t>a$. Also assume that $f^{\Delta}(t, \cdot)$ is rd-continuous on $[a, \sigma(t)]$. Suppose that for each $\varepsilon>0$ there is a neighborhood $U$ of $t$, independent of $\tau \in[a, \sigma(t)]$, such that

$$
\begin{aligned}
& \left|[f(\sigma(t), \tau)-f(s, \tau)]-f^{\Delta}(t, \tau)[\sigma(t)-s]\right| \\
& \quad \leq \varepsilon|\sigma(t)-s|
\end{aligned}
$$

for all $s \in U$, where $f^{\Delta}$ denotes the derivative of $f$ with respect to the first variable. Then

(i) $g(t):=\int_{a}^{t} f(t, \tau) \Delta \tau$ implies $g^{\Delta}(t):=\int_{a}^{t} f^{\Delta}(t, \tau) \Delta \tau+$ $f(\sigma(t), t)$;

(ii) $g(t):=\int_{t}^{b} f(t, \tau) \Delta \tau$ implies $g^{\Delta}(t):=\int_{t}^{b} f^{\Delta}(t, \tau) \Delta \tau-$ $f(\sigma(t), t)$.

A function $p: \mathbb{T} \longrightarrow \mathbb{R}$ is said to be regressive provided $1+\mu(t) p(t) \neq 0, \forall t \in \mathbb{T}^{\kappa}$ and is said to be positive regressive provided $1+\mu(t) p(t)>0, \forall t \in \mathbb{T}^{\kappa}$. The set of all regressive 
and rd-continuous functions $p: \mathbb{T} \longrightarrow \mathbb{R}$ are denoted by $\mathscr{R}=\mathscr{R}(\mathbb{T})=\mathscr{R}(\mathbb{T}, \mathbb{R})$ and the set of all positive regressive and rd-continuous functions $p: \mathbb{T} \longrightarrow \mathbb{R}$ are denoted by $\mathscr{R}^{+}=\mathscr{R}^{+}(\mathbb{T}, \mathbb{R})=\{p \in \mathscr{R}: 1+\mu(t) p(t)>0, \forall t \in \mathbb{T}\}$.

If $p \in \mathscr{R}^{+}$, then the exponential function is defined by

$$
e_{p}(t, s)=\exp \left(\int_{s}^{t} \xi_{\mu(\tau)}(p(\tau)) \Delta \tau\right), \quad \forall t, s \in \mathbb{T},
$$

with the cylinder transformation

$$
\xi_{h}(z)= \begin{cases}\frac{\log (1+h z)}{h}, & \text { if } h \neq 0, \\ z, & \text { if } h=0\end{cases}
$$

Let $p, q: \mathbb{T} \longrightarrow \mathbb{R}$ be two regressive functions; define

$$
\begin{gathered}
p \oplus q=p+q+\mu p q, \\
\ominus p=-\frac{p}{1+\mu p}, \\
p \ominus q=p \oplus(\ominus q) .
\end{gathered}
$$

Lemma 2 (see [36]). If $p \in \mathscr{R}^{+}$, then

(i) $e_{p}(t, s)>0$, for all $t, s \in \mathbb{T}$;

(ii) if $p(t) \leq q(t)$, then $e_{p}(t, s) \leq e_{q}(t, s)$ for all $t \geq s, t, s \in$ $\mathbb{T}$.

Definition 3 (see [46]). We say that a time scale $\mathbb{T}$ is periodic if there exists $p>0$ such that if $t \in \mathbb{T}$, then $t \pm p \in \mathbb{T}$. For $\mathbb{\square} \neq \mathbb{R}$, the smallest positive $p$ is called the period of the time scale.

Definition 4 (see [46]). Let $\mathbb{T} \neq \mathbb{R}$ be a periodic time scale with period $p$. We say that the function $f: \mathbb{T} \rightarrow \mathbb{R}$ is periodic with period $\omega$ if there exists a natural number $n$ such that $\omega=n p, f(t+\omega)=f(t)$ for all $t \in \mathbb{T}$ and $\omega$ is the smallest positive number such that $f(t+\omega)=f(t)$.

Remark 5. From [47], we know that if $\mathbb{T}$ is $\omega$-periodic, then the graininess function $\mu$ is $\omega$-periodic.

Lemma 6 (see [47]). Let $\mathbb{T}$ be $\omega$-periodic and suppose $f: \mathbb{T} \times$ $\mathbb{T} \longrightarrow \mathbb{R}$ satisfies the assumptions of Lemma 1 . Define $g(t)=$ $\int_{t}^{t+\omega} f(t, s) \Delta s$. If $f^{\Delta}(t, s)$ denotes the derivative of $f$ with respect to $t$, then

$$
\begin{aligned}
g^{\Delta}(t)= & \int_{t}^{t+\omega} f^{\Delta}(t, s) \Delta s+f(\sigma(t), t+\omega) \\
& -f(\sigma(t), t) .
\end{aligned}
$$

Definition 7. Let $\mathbb{T}$ be an $\omega$-periodic time scale. A function $u$ : $\mathbb{T} \longrightarrow \mathbb{R}$ is said to be $\omega$-anti-periodic on $\mathbb{T}$ if $u(t+\omega)=-u(t)$ for all $t \in \mathbb{T}, \omega>0$ is a constant.

Definition 8. Let $\mathbb{T}$ be an $\omega$-periodic time scale. A function $f=f^{R}+f^{I} i+f^{J} j+f^{K} k: \mathbb{T} \longrightarrow \mathbb{Q}^{n}$ is called an $\omega$-antiperiodic, where $f^{m}: \mathbb{T} \longrightarrow \mathbb{R}^{n}, m \in\{R, I, J, K\}:=M$ if for every $m \in M, f^{m}$ is $\omega$-anti-periodic.
In this paper, we consider the following QVFCNN with time-varying delays on time scales:

$$
\begin{aligned}
x_{l}^{\Delta}(t)= & -a_{l}(t) x_{l}(t)+\sum_{h=1}^{n} b_{l h}(t) f_{h}\left(x_{h}\left(t-\tau_{l h}(t)\right)\right) \\
& +\sum_{h=1}^{n} d_{l h}(t) \mu_{h}(t) \\
& +\bigwedge_{h=1}^{n} \alpha_{l h}(t) \int_{t-\eta_{l h}(t)}^{t} g_{h}\left(x_{h}(s)\right) \Delta s \\
& +\bigvee_{h=1}^{n} \beta_{l h}(t) \int_{t-\xi_{l h}(t)}^{t} g_{h}\left(x_{h}(s)\right) \Delta s \\
& +\bigwedge_{h=1}^{n} T_{l h}(t) \mu_{h}(t)+\bigvee_{h=1}^{n} S_{l h}(t) \mu_{h}(t)+I_{l}(t),
\end{aligned}
$$

where $l \in\{1,2, \ldots, n\}:=\Lambda, t \in \mathbb{T}, \mathbb{T}$ is a periodic time scale; $n$ is the number of neurons in layers; $x_{l}(t) \in \mathbb{Q}$ and $\mu_{h}(t) \in \mathbb{Q}$ are the state of the $l$ th neuron at time $t$ and the deviations of the $h$ th neuron at time $t$, respectively; $a_{l}(t)>$ 0 represents the rate with which the $l$ th neuron will reset its potential to the resting state in isolation when they are disconnected from the network and the external inputs at time $t, \alpha_{l h}(t) \in \mathbb{Q}, \beta_{l h}(t) \in \mathbb{Q}, T_{l h}(t) \in \mathbb{Q}$, and $S_{l h}(t) \in \mathbb{Q}$ are the elements of fuzzy feedback MIN template, fuzzy feedback MAX template, fuzzy feed forward MIN template, and fuzzy feed forward MAX template, respectively; $b_{l h}(t) \in \mathbb{Q}$ and $d_{l h}(t) \in \mathbb{Q}$ are the elements of feedback template and feed forward template, $\bigwedge, \bigvee$ denote the fuzzy AND and fuzzy OR operations, respectively; $f_{h}(\cdot)$ and $g_{h}(\cdot): \mathbb{Q} \longrightarrow \mathbb{Q}$ are the activation functions; $\tau_{l h}(t) \geq 0, \eta_{l h}(t) \geq 0$ and $\xi_{l h}(t) \geq 0$ correspond to transmission delays at time $t$ and satisfy $t-\tau_{l h}(t) \in \mathbb{T}, t-\eta_{l h}(t) \in \mathbb{T}, t-\xi_{l h}(t) \in \mathbb{T}$ for $t \in \mathbb{T}$; $I_{l}(t) \in \mathbb{Q}$ denotes the input of the $l$ th neuron at time $t, l \in \Lambda$.

Throughout the rest of the paper, we denote $[a, b]_{\mathbb{T}}=\{t \mid$ $t \in[a, b] \cap \mathbb{T}\}$. For convenience, for a bounded and continuous function $f: \mathbb{T} \longrightarrow \mathbb{R}$, we denote $f=\inf _{t \in \mathbb{T}}|f(t)|$ and $\bar{f}=$ $\sup _{t \in \mathbb{T}}|f(t)|$.

The initial conditions of system (10) are

$$
x_{l}(s)=\varphi_{l}(s), \quad s \in\left[t_{0}-v, t_{0}\right]_{\mathbb{T}},
$$

where $v=\max _{l, h \in I}\left\{\bar{\tau}_{l h}, \bar{\eta}_{l h}, \bar{\xi}_{l h}\right\}, \varphi_{l} \in C_{r d}\left(\left[t_{0}-v, t_{0}\right]_{\mathbb{T}}, \mathbb{Q}\right)$, $l \in \Lambda$.

Lemma 9 (see [8]). Suppose $x$ and $y$ are two states of system (15). Then we have

$$
\begin{aligned}
& \left|\bigwedge_{j=1}^{n} \alpha_{i j}(t) f_{j}(x)-\bigwedge_{j=1}^{n} \alpha_{i j}(t) f_{j}(y)\right| \\
& \quad \leq \sum_{j=1}^{n}\left|\alpha_{i j}(t)\right|\left|f_{j}(x)-f_{j}(y)\right|, \quad i \in \Lambda,
\end{aligned}
$$




$$
\begin{aligned}
& \left|\bigvee_{j=1}^{n} \beta_{i j}(t) f_{j}(x)-\bigvee_{j=1}^{n} \beta_{i j}(t) f_{j}(y)\right| \\
& \quad \leq \sum_{j=1}^{n}\left|\beta_{i j}(t)\right|\left|f_{j}(x)-f_{j}(y)\right|, \quad i \in \Lambda .
\end{aligned}
$$$$
+\bigwedge_{h=1}^{n} T_{l h}^{*}(t) U_{h}(t)+\bigvee_{h=1}^{n} S_{l h}^{*}(t) U_{h}(t)+I_{l}^{*}(t)
$$$$
l \in \Lambda, t \in \mathbb{T},
$$

(12)

In order to overcome the inconvenience of the noncommutativity of quaternion multiplication, in the following, we will first decompose system (10) into the vector form of the four real-valued systems. To do so, for $x_{h}=x_{h}^{R}+x_{h}^{I} i+$ $x_{h}^{J} j+x_{h}^{K} k \in \mathbb{Q}$, where $x_{h}^{R}, x_{h}^{I}, x_{h}^{J}, x_{h}^{K} \in \mathbb{R}$, we assume that the activation functions $f_{h}\left(x_{h}\right)$ and $g_{h}\left(x_{h}\right)$ of (10) can be expressed as

$$
\begin{aligned}
f_{h}\left(x_{h}\right)= & f_{h}^{R}\left(x_{h}^{R}, x_{h}^{I}, x_{h}^{J}, x_{h}^{K}\right)+i f_{h}^{I}\left(x_{h}^{R}, x_{h}^{I}, x_{h}^{J}, x_{h}^{K}\right) \\
& +j f_{h}^{J}\left(x_{h}^{R}, x_{h}^{I}, x_{h}^{J}, x_{h}^{K}\right) \\
& +k f_{h}^{K}\left(x_{h}^{R}, x_{h}^{I}, x_{h}^{J}, x_{h}^{K}\right), \\
g_{h}\left(x_{h}\right)= & g_{h}^{R}\left(x_{h}^{R}, x_{h}^{I}, x_{h}^{J}, x_{h}^{K}\right)+i g_{h}^{I}\left(x_{h}^{R}, x_{h}^{I}, x_{h}^{J}, x_{h}^{K}\right) \\
& +j g_{h}^{J}\left(x_{h}^{R}, x_{h}^{I}, x_{h}^{J}, x_{h}^{K}\right) \\
& +k g_{h}^{K}\left(x_{h}^{R}, x_{h}^{I}, x_{h}^{J}, x_{h}^{K}\right),
\end{aligned}
$$

where $f_{h}^{m}, g_{h}^{m}: \mathbb{R}^{4} \longrightarrow \mathbb{R}, h \in \Lambda, m \in M$.

Remark 10. In system (10), if $x_{h}=x_{h}^{R}+x_{h}^{I} i \in \mathbb{C}$, where $x_{h}^{R}, x_{h}^{I} \in \mathbb{R}$ and the activation functions $f_{h}, g_{h}: \mathbb{C} \longrightarrow \mathbb{C}$ are complex variable functions, that is,

$$
\begin{aligned}
& f_{h}\left(x_{h}\right)=f_{h}^{R}\left(x_{h}^{R}, x_{h}^{I}\right)+i f_{h}^{I}\left(x_{h}^{R}, x_{h}^{I}\right), \\
& g_{h}\left(x_{h}\right)=g_{h}^{R}\left(x_{h}^{R}, x_{h}^{I}\right)+i g_{h}^{I}\left(x_{h}^{R}, x_{h}^{I}\right),
\end{aligned}
$$

where $f_{h}^{m}, g_{h}^{m}: \mathbb{R}^{2} \longrightarrow \mathbb{R}, h \in \Lambda, m=R, I$, and all the quaternion-valued coefficients of (10) are transformed to the complex-valued coefficients, then system (10) degenerates to a complex-valued system; if all of the activation functions and coefficients of (10) are real variable functions, then system (10) degenerates to a real-valued system.

It follows from Hamilton's multiplication rules that the equivalent real-valued system of QVFCNN (10) can be written as follows:

$$
\begin{aligned}
X_{l}^{\Delta}(t)= & -a_{l}(t) X_{l}(t)+\sum_{h=1}^{n} B_{l h}(t) \widetilde{F}_{h}\left[x_{h}\left(t-\tau_{l h}(t)\right)\right] \\
& +\sum_{h=1}^{n} D_{l h}(t) U_{h}(t) \\
& +\bigwedge_{h=1}^{n} A_{l h}(t) \int_{t-\eta_{l h}(t)}^{t} \widetilde{G}_{h}\left[x_{h}(s)\right] \Delta s \\
& +\bigvee_{h=1}^{n} C_{l h}(t) \int_{t-\xi_{l h}(t)}^{t} \widetilde{G}_{h}\left[x_{h}(s)\right] \Delta s
\end{aligned}
$$

where

$$
\begin{aligned}
& \tilde{f}_{h}^{m}\left[x_{h}\right]=f_{h}^{m}\left(x_{h}^{R}, x_{h}^{I}, x_{h}^{J}, x_{h}^{K}\right), \\
& \tilde{g}_{h}^{m}\left[x_{h}\right]=g_{l}^{m}\left(x_{h}^{R}, x_{h}^{I}, x_{h}^{J}, x_{h}^{K}\right), \\
& X_{l}(t)=\left(\begin{array}{c}
x_{l}^{R}(t) \\
x_{l}^{I}(t) \\
x_{l}^{J}(t) \\
x_{l}^{K}(t)
\end{array}\right), \\
& I_{l}^{*}(t)=\left(\begin{array}{c}
I_{l}^{R}(t) \\
I_{l}^{I}(t) \\
I_{l}^{J}(t) \\
I_{l}^{K}(t)
\end{array}\right),
\end{aligned}
$$$$
\widetilde{G}_{h}\left[x_{h}(t)\right]=\left(\begin{array}{c}
\tilde{g}_{h}^{R}\left[x_{h}(t)\right] \\
\tilde{g}_{h}^{I}\left[x_{h}(t)\right] \\
\tilde{g}_{h}^{J}\left[x_{h}(t)\right] \\
\tilde{g}_{h}^{K}\left[x_{h}(t)\right]
\end{array}\right),
$$$$
U_{h}(t)=\left(\begin{array}{c}
\mu_{h}^{R}(t) \\
\mu_{h}^{I}(t) \\
\mu_{h}^{J}(t) \\
\mu_{h}^{K}(t)
\end{array}\right)
$$$$
B_{l h}(t)=\left(\begin{array}{cccc}
b_{l h}^{R}(t) & -b_{l h}^{I}(t) & -b_{l h}^{J}(t) & -b_{l h}^{K}(t) \\
b_{l h}^{I}(t) & b_{l h}^{R}(t) & -b_{l h}^{K}(t) & b_{l h}^{J}(t) \\
b_{l h}^{J}(t) & b_{l h}^{K}(t) & b_{l h}^{R}(t) & -b_{l h}^{I}(t) \\
b_{l h}^{K}(t) & -b_{l h}^{J}(t) & b_{l h}^{I}(t) & b_{l h}^{R}(t)
\end{array}\right),
$$$$
D_{l h}(t)=\left(\begin{array}{cccc}
d_{l h}^{R}(t) & -d_{l h}^{I}(t) & -d_{l h}^{J}(t) & -d_{l h}^{K}(t) \\
d_{l h}^{I}(t) & d_{l h}^{R}(t) & -d_{l h}^{K}(t) & d_{l h}^{J}(t) \\
d_{l h}^{J}(t) & d_{l h}^{K}(t) & d_{l h}^{R}(t) & -d_{l h}^{I}(t) \\
d_{l h}^{K}(t) & -d_{l h}^{J}(t) & d_{l h}^{I}(t) & d_{l h}^{R}(t)
\end{array}\right),
$$$$
A_{l h}(t)=\left(\begin{array}{cccc}
\alpha_{l h}^{R}(t) & -\alpha_{l h}^{I}(t) & -\alpha_{l h}^{J}(t) & -\alpha_{l h}^{K}(t) \\
\alpha_{l h}^{I}(t) & \alpha_{l h}^{R}(t) & -\alpha_{l h}^{K}(t) & \alpha_{l h}^{J}(t) \\
\alpha_{l h}^{J}(t) & \alpha_{l h}^{K}(t) & \alpha_{l h}^{R}(t) & -\alpha_{l h}^{I}(t) \\
\alpha_{l h}^{K}(t) & -\alpha_{l h}^{J}(t) & \alpha_{l h}^{I}(t) & \alpha_{l h}^{R}(t)
\end{array}\right),
$$ 


$$
\begin{aligned}
& C_{l h}(t)=\left(\begin{array}{cccc}
\beta_{l h}^{R}(t) & -\beta_{l h}^{I}(t) & -\beta_{l h}^{I}(t) & -\beta_{l h}^{K}(t) \\
\beta_{l h}^{I}(t) & \beta_{l h}^{R}(t) & -\beta_{l h}^{K}(t) & \beta_{l h}^{I}(t) \\
\beta_{l h}^{J}(t) & \beta_{l h}^{K}(t) & \beta_{l h}^{R}(t) & -\beta_{l h}^{I}(t) \\
\beta_{l h}^{K}(t) & -\beta_{l h}^{I}(t) & \beta_{l h}^{I}(t) & \beta_{l h}^{R}(t)
\end{array}\right), \\
& S_{l h}^{*}(t)=\left(\begin{array}{cccc}
S_{l h}^{R}(t) & -S_{l h}^{I}(t) & -S_{l h}^{I}(t) & -S_{l h}^{K}(t) \\
S_{l h}^{I}(t) & S_{l h}^{R}(t) & -S_{l h}^{K}(t) & S_{l h}^{I}(t) \\
S_{l h}^{J}(t) & S_{l h}^{K}(t) & S_{l h}^{R}(t) & -S_{l h}^{I}(t) \\
S_{l h}^{K}(t) & -S_{l h}^{I}(t) & S_{l h}^{I}(t) & S_{l h}^{R}(t)
\end{array}\right), \\
& T_{l h}^{*}(t)=\left(\begin{array}{cccc}
T_{l h}^{R}(t) & -T_{l h}^{I}(t) & -T_{l h}^{J}(t) & -T_{l h}^{K}(t) \\
T_{l h}^{I}(t) & T_{l h}^{R}(t) & -T_{l h}^{K}(t) & T_{l h}^{J}(t) \\
T_{l h}^{J}(t) & T_{l h}^{K}(t) & T_{l h}^{R}(t) & -T_{l h}^{I}(t) \\
T_{l h}^{K}(t) & -T_{l h}^{J}(t) & T_{l h}^{I}(t) & T_{l h}^{R}(t)
\end{array}\right), \\
& \widetilde{F}_{h}\left[x_{h}\left(t-\tau_{l h}(t)\right)\right]=\left(\begin{array}{c}
\tilde{f}_{h}^{R}\left[x_{h}\left(t-\tau_{l h}(t)\right)\right] \\
\tilde{f}_{h}^{I}\left[x_{h}\left(t-\tau_{l h}(t)\right)\right] \\
\tilde{f}_{h}^{J}\left[x_{h}\left(t-\tau_{l h}(t)\right)\right] \\
\tilde{f}_{h}^{K}\left[x_{h}\left(t-\tau_{l h}(t)\right)\right]
\end{array}\right),
\end{aligned}
$$

with the initial conditions

$$
X_{l}(s)=\Phi_{l}(s), \quad s \in\left[t_{0}-v, t_{0}\right]_{\mathbb{T}},
$$

where $\Phi_{l}(s)=\left(\varphi_{l}^{R}(s), \varphi_{l}^{I}(s), \varphi_{l}^{J}(s), \varphi_{l}^{K}(s)\right)^{T}, \varphi_{l}^{m}(s) \in C_{r d}\left(\left[t_{0}-\right.\right.$ $\left.\left.\nu, t_{0}\right]_{\mathbb{T}}, \mathbb{R}\right), l \in \Lambda, m \in M$.

Remark 11. It is easy to see that if $X(t)=$ $\left(X_{1}(t), X_{2}(t), \ldots, X_{n}(t)\right)^{T}$ is a solution of system (15), where $X_{l}(t)=\left(x_{l}^{R}(t), x_{l}^{I}(t), x_{l}^{J}(t), x_{l}^{K}(t)\right), l \in \Lambda$, then $x(t)=\left(x_{1}(t), x_{2}(t), \ldots, x_{n}(t)\right)^{T}$, where $x_{l}(t)=$ $x_{l}^{R}(t)+i x_{l}^{I}(t)+j x_{l}^{J}(t)+k x_{l}^{K}(t), l \in \Lambda$ is a solution of (10). Thus, the problem of finding an anti-periodic solution for (10) is reduced to finding one for system (15). For considering the stability of anti-periodic solutions of (10), we just need to consider the stability of anti-periodic solutions of system (15).

\section{The Existence of Anti-Periodic Solutions}

In this section, we will state and prove the sufficient conditions for the existence of anti-periodic solutions of (15).

Let $\mathbb{X}=\left\{\varphi \mid \varphi=\left(\varphi_{1}^{R}, \varphi_{1}^{I}, \varphi_{1}^{J}, \varphi_{1}^{K}, \varphi_{2}^{R}, \varphi_{2}^{I}, \varphi_{2}^{J}, \varphi_{2}^{K}, \ldots, \varphi_{n}^{R}\right.\right.$, $\left.\varphi_{n}^{I}, \varphi_{n}^{J}, \varphi_{n}^{K}\right)^{T}:=\left(\varphi_{1}, \varphi_{2}, \ldots, \varphi_{n}\right)^{T} \in C\left(\mathbb{T}, \mathbb{R}^{4 n}\right) \quad:$ $\varphi(t+\omega)=-\varphi(t), \forall t \in \mathbb{T}\}$ with the norm $\|\varphi\|_{\mathbb{X}}=$ $\max \left\{\max _{l \in \Lambda, m \in M}\left\{\sup _{t \in \mathbb{T}}\left|\varphi_{l}^{m}(t)\right|\right\}\right\}$, then $\mathbb{X}$ is a Banach space. Let $\varphi^{0}=\left(\left(\varphi_{1}^{0}\right)^{R},\left(\varphi_{1}^{0}\right)^{I},\left(\varphi_{1}^{0}\right)^{J},\left(\varphi_{1}^{0}\right)^{K},\left(\varphi_{2}^{0}\right)^{R},\left(\varphi_{2}^{0}\right)^{I},\left(\varphi_{2}^{0}\right)^{J},\left(\varphi_{2}^{0}\right)^{K}\right.$, $\left.\ldots,\left(\varphi_{n}^{0}\right)^{R},\left(\varphi_{n}^{0}\right)^{I},\left(\varphi_{n}^{0}\right)^{J},\left(\varphi_{n}^{0}\right)^{K}\right)^{T}:=\left(\varphi_{1}^{0}, \varphi_{2}^{0}, \ldots, \varphi_{n}^{0}\right)^{T}$ where $\varphi_{l}^{0}(t)=\int_{t}^{t+\omega} G_{-a_{l}}(t, \sigma(s))\left(\sum_{h=1}^{n} D_{l h}(s) \times U_{h}(s)+\right.$ $\left.\bigwedge_{h=1}^{n} T_{l h}^{*}(s) U_{h}(s)+\bigvee_{h=1}^{n} S_{l h}^{*}(s) U_{h}(s)+I_{l}^{*}(s)\right) \Delta s, l \in \Lambda$ and $r$ be a constant satisfying $r \geq\left\|\varphi^{0}\right\|_{\mathbb{X}}$.
Throughout this paper, we assume that the following conditions hold:

$\left(H_{1}\right)$ Functions $a_{l}, \tau_{l h}, \eta_{l h}, \xi_{l h} \in C\left(\mathbb{T}, \mathbb{R}^{+}\right)$with $-a_{l} \in \mathscr{R}^{+}$, $b_{l h}^{m}, d_{l h}^{m}, \alpha_{l h}^{m}, \beta_{l h}^{m}, T_{l h}^{m}, S_{l h}^{m} \in C(\mathbb{T}, \mathbb{R})$ are $\omega$-periodic, $\mu_{h}^{m}, I_{l}^{m} \in C(\mathbb{T}, \mathbb{R})$ are $\omega$-anti-periodic, and functions $f_{h}^{m}, g_{h}^{m} \in C\left(\mathbb{R}^{4}, \mathbb{R}\right)$ satisfy

$$
\begin{aligned}
& f_{h}^{m}\left(x_{h}^{R}, x_{h}^{I}, x_{h}^{J}, x_{h}^{K}\right)=-f_{h}^{m}\left(-x_{h}^{R},-x_{h}^{I},-x_{h}^{J},-x_{h}^{K}\right), \\
& g_{l}^{m}\left(x_{h}^{R}, x_{h}^{I}, x_{h}^{J}, x_{h}^{K}\right)=-g_{l}^{m}\left(-x_{h}^{R},-x_{h}^{I},-x_{h}^{J},-x_{h}^{K}\right),
\end{aligned}
$$

where $x_{l}^{m} \in \mathbb{R}, l, h \in \Lambda, m \in M$.

$\left(H_{2}\right)$ Functions $f_{h}^{m}, g_{h}^{m} \in C\left(\mathbb{R}^{4}, \mathbb{R}\right)$ and there exist positive constants $L_{h}^{m}, H_{h}^{m}$ such that for all $x_{h}^{m}, y_{h}^{m} \in \mathbb{R}$,

$$
\begin{aligned}
& \left|f_{h}^{m}\left(x_{h}^{R}, x_{h}^{I}, x_{h}^{J}, x_{h}^{K}\right)-f_{h}^{m}\left(y_{h}^{R}, y_{h}^{I}, y_{h}^{J}, y_{h}^{K}\right)\right| \\
& \leq L_{h}^{R}\left|x^{R}-y^{R}\right|+L_{h}^{I}\left|x^{I}-y^{I}\right|+L_{h}^{J}\left|x^{J}-y^{J}\right| \\
& \quad+L_{h}^{K}\left|x^{K}-y^{K}\right|, \\
& \left|g_{h}^{m}\left(x_{h}^{R}, x_{h}^{I}, x_{h}^{J}, x_{h}^{K}\right)-g_{h}^{m}\left(y_{h}^{R}, y_{h}^{I}, y_{h}^{J}, y_{h}^{K}\right)\right| \\
& \leq H_{h}^{R}\left|x^{R}-y^{R}\right|+H_{h}^{I}\left|x^{I}-y^{I}\right|+H_{h}^{J}\left|x^{J}-y^{J}\right| \\
& \quad+H_{h}^{K}\left|x^{K}-y^{K}\right|
\end{aligned}
$$

and $f_{h}^{m}(0,0,0,0)=0, g_{h}^{m}(0,0,0,0)=0, h \in \Lambda, m \in$ M.

$\left(H_{3}\right)$

$$
\max _{l \in \Lambda}\left\{\frac{\omega \kappa_{l} P_{l}}{1+\kappa_{l}}\right\}:=\xi<1,
$$

where $\kappa_{l}=e_{-a_{l}}(0, \omega), l \in \Lambda$,

$$
\begin{aligned}
P_{l}= & \sum_{h=1}^{n} L_{h}\left(\bar{b}_{l h}^{R}+\bar{b}_{l h}^{I}+\bar{b}_{l h}^{J}+\bar{b}_{l h}^{K}\right) \\
& +\sum_{h=1}^{n} \bar{\eta}_{l h} H_{h}\left(\bar{\alpha}_{l h}^{R}+\bar{\alpha}_{l h}^{I}+\bar{\alpha}_{l h}^{I}+\bar{\alpha}_{l h}^{K}\right) \\
& +\sum_{h=1}^{n} \bar{\xi}_{l h} H_{h}\left(\bar{\beta}_{l h}^{R}+\bar{\beta}_{l h}^{I}+\bar{\beta}_{l h}^{I}+\bar{\beta}_{l h}^{K}\right), \quad l \in \Lambda, \\
L_{h}= & L_{h}^{R}+L_{h}^{I}+L_{h}^{J}+L_{h}^{K}, \\
H_{h}= & H_{h}^{R}+H_{h}^{I}+H_{h}^{J}+H_{h}^{K}, \quad h \in \Lambda .
\end{aligned}
$$

Lemma 12. Suppose that $\left(H_{1}\right)$ holds. Then $X(t)=$ $\left(X_{1}(t), X_{2}(t), \ldots, X_{n}(t)\right)^{T}$ is an $\omega$-anti-periodic solution of system (15) if and only if $X_{l}(t)$ satisfies

$$
X_{l}(t)=\int_{t}^{t+\omega} G_{l}(t, \sigma(s)) K_{l}(s, x) \Delta s, \quad l \in \Lambda, t \in \mathbb{T},
$$


where $G_{l}(t, \sigma(s))=-e_{-a_{l}}(t, \sigma(s)) /\left(1+e_{-a_{l}}(0, \omega)\right)$,

$$
\begin{aligned}
K_{l}(s, x)= & \sum_{h=1}^{n} B_{l h}(s) \widetilde{F}_{h}\left[x_{h}\left(s-\tau_{l h}(s)\right)\right] \\
& +\sum_{h=1}^{n} D_{l h}(s) U_{h}(s) \\
& +\bigwedge_{h=1}^{n} A_{l h}(s) \int_{s-\eta_{l h}(s)}^{s} \widetilde{G}_{h}\left[x_{h}(u)\right] \Delta u \\
& +\bigvee_{h=1}^{n} C_{l h}(s) \int_{s-\xi_{l h}(s)}^{s} \widetilde{G}_{h}\left[x_{h}(u)\right] \Delta u \\
& +\bigwedge_{h=1}^{n} T_{l h}^{*}(s) U_{h}(s)+\bigvee_{h=1}^{n} S_{l h}^{*}(s) U_{h}(s) \\
& +I_{l}^{*}(s) .
\end{aligned}
$$

Proof. Let $X(t)$ be an $\omega$-anti-periodic solution of system (15). Set $Y_{l}(s)=e_{-a_{l}}(t, s) X_{l}(s)$, then

$$
\begin{aligned}
Y_{l}^{\Delta}(s)= & a_{l}(s) e_{-a_{l}}(t, \sigma(s)) X_{l}(s) \\
& +e_{-a_{l}}(t, \sigma(s)) X_{l}^{\Delta}(s) \\
= & e_{-a_{l}}(t, \sigma(s))\left(a_{l}(s) X_{l}(s)+X_{l}^{\Delta}(s)\right) \\
= & e_{-a_{l}}(t, \sigma(s)) K_{l}(s, x) .
\end{aligned}
$$

Integrating (24) from $t$ to $t+\omega$, we have

$$
Y(t+\omega)-Y(t)=\int_{t}^{t+\omega} e_{-a_{l}}(t, \sigma(s)) K_{l}(s, x) \Delta s .
$$

Note that $Y_{l}(t)=X_{l}(t)$, thus

$$
\begin{aligned}
& e_{-a_{l}}(t, t+\omega) X_{l}(t+\omega) \\
& =X_{l}(t)+\int_{t}^{t+\omega} e_{-a_{l}}(t, \sigma(s)) K_{l}(s, x) \Delta s .
\end{aligned}
$$

Since $X_{l}(t+\omega)=-X_{l}(t)$, we have

$$
X_{l}(t)=\int_{t}^{t+\omega} G_{l}(t, \sigma(s)) K_{l}(s, x) \Delta s, \quad l \in \Lambda, t \in \mathbb{T},
$$

that is, $X(t)$ is a solution of (22).

On the other hand, let $X(t)$ be a solution of (22). By Lemma 6, we obtain

$$
X_{l}^{\Delta}(t)=\left(-\int_{t}^{t+\omega} \frac{e_{-a_{l}}(t, \sigma(s))}{1+e_{-a_{l}}(0, \omega)} K_{l}(s, x) \Delta s\right)^{\Delta}
$$

$$
\begin{aligned}
& =\int_{t}^{t+\omega} \frac{a_{l}(t) e_{-a_{l}}(t, \sigma(s))}{1+e_{-a_{l}}(0, \omega)} K_{l}(s, x) \Delta s \\
& -\frac{e_{-a_{l}}(\sigma(t), \sigma(t)+\omega)}{1+e_{-a_{l}}(0, \omega)} K_{l}(t+\omega, x) \\
& +\frac{e_{-a_{l}}(\sigma(t), \sigma(t))}{1+e_{-a_{l}}(0, \omega)} K_{l}(t, x) \\
& =\int_{t}^{t+\omega} \frac{a_{l}(t) e_{-a_{l}}(t, \sigma(s))}{1+e_{-a_{l}}(0, \omega)}\left(X_{l}^{\Delta}(s)\right. \\
& \left.+a_{l}(s) X_{l}(s)\right) \Delta s-\frac{e_{-a_{l}}(\sigma(t), \sigma(t)+\omega)}{1+e_{-a_{l}}(0, \omega)} \\
& \cdot K_{l}(t+\omega, x)+\frac{e_{-a_{l}}(\sigma(t), \sigma(t))}{1+e_{-a_{l}}(0, \omega)} K_{l}(t, x) \\
& =\frac{a_{l}(t)}{1+e_{-a_{l}}(0, \omega)} \int_{t}^{t+\omega} e_{-a_{l}}(t, \sigma(s))\left(X_{l}^{\Delta}(s)\right. \\
& \left.+a_{l}(s) X_{l}(s)\right) \Delta s+K_{l}(t, x)=\frac{a_{l}(t)}{1+e_{-a_{l}}(0, \omega)} \\
& \cdot \int_{t}^{t+\omega}\left(e_{-a_{l}}(t, s) X_{l}(s)\right)^{\Delta} \Delta s+K_{l}(t, x)=-a_{l}(t) \\
& \cdot X_{l}(t)+K_{l}(t, x), \quad l \in \Lambda, t \in \mathbb{T} .
\end{aligned}
$$

Therefore, $\left(X_{1}(t), X_{2}(t), \ldots, X_{n}(t)\right)^{T}$ is an $\omega$-anti-periodic solution of system (15). The proof of Lemma 12 is completed.

Remark 13. It is easy to see that

$$
\begin{aligned}
& \frac{1}{1+\kappa_{l}} \leq\left|G_{l}(t, \sigma(s))\right| \leq \frac{\kappa_{l}}{1+\kappa_{l}}, \\
& \sigma(s) \in[t, t+\omega]_{\mathbb{T}},
\end{aligned}
$$

$l \in \Lambda, t \in \mathbb{T}$.

Theorem 14. Let $\left(H_{1}\right)-\left(H_{3}\right)$ hold. Then, system (15) has a unique $\omega$-anti-periodic solution in the region $\mathbb{X}^{*}=\{\varphi \in \mathbb{X}$ : $\left.\left\|\varphi-\varphi^{0}\right\|_{\mathbb{X}} \leq \xi r /(1-\xi)\right\}$.

Proof. Define an operator $\Phi: \mathbb{X}^{*} \longrightarrow \mathbb{X}$ by

$$
\begin{aligned}
\Phi \varphi & =\left((\Phi \varphi)_{1},(\Phi \varphi)_{2}, \ldots,(\Phi \varphi)_{n}\right)^{T} \\
& \longrightarrow\left(X_{1}^{\varphi}, X_{2}^{\varphi}, \ldots, X_{n}^{\varphi}\right)^{T}
\end{aligned}
$$

where $X_{l}^{\varphi}(t)=\int_{t}^{t+\omega} G_{l}(t, \sigma(s)) K_{l}(s, \varphi) \Delta s, t \in \mathbb{T}, l \in \Lambda$ and $G_{l}, K_{l}$ are defined the same as those in Lemma 12.

For $\varphi \in \mathbb{X}^{*}$, then $\|\varphi\|_{\mathbb{X}} \leq\left\|\varphi-\varphi^{0}\right\|_{\mathbb{X}}+\left\|\varphi^{0}\right\|_{\mathbb{X}} \leq \xi r /(1-\xi)+$ $r=r /(1-\xi)$. First, we show that for any $\varphi \in \mathbb{X}^{*}, \Phi \varphi \in \mathbb{X}^{*}$. From $\left(\mathrm{H}_{2}\right)$, we have 
$=\left\{\int_{t}^{t+\omega}\left[G_{l}(t+\omega, \sigma(s)+\omega)\right.\right.$

$\left.\left.\cdot\left(\sum_{h=1}^{n} B_{l h}(s+\omega) \tilde{F}_{h}\left[\varphi_{h}\left(s+\omega-\tau_{l h}(s+\omega)\right)\right]+\sum_{h=1}^{n} D_{l h}(s+\omega) U_{h}(s+\omega)+\bigwedge_{h=1}^{n} A_{l h}(s+\omega) \int_{s+\omega-\eta_{h l}(s+\omega)}^{s+\omega} \widetilde{G}_{h}\left[\varphi_{h}(u)\right] \Delta u+\bigvee_{h=1}^{n} C_{l h}(s+\omega) \int_{s+\omega-\xi_{h l}(s+\omega)}^{s+\omega} \widetilde{G}_{h}\left[\varphi_{h}(u)\right] \Delta u+\bigwedge_{h=1}^{n} T_{h h}^{*}(s+\omega) U_{h}(s+\omega)+\bigvee_{h=1}^{n} s_{l h}^{*}(s+\omega) U_{h}(s+\omega)+I_{l}^{*}(s+\omega)\right)\right] \Delta s\right\}$ $=-\left\{\int_{t}^{t+\omega}\left[G_{l}(t, \sigma(s))\left(\sum_{h=1}^{n} B_{l h}(s) \tilde{F}_{h}\left[\varphi_{h}\left(s-\tau_{l h}(s)\right)\right]+\sum_{h=1}^{n} D_{l h}(s) U_{h}(s)+\bigwedge_{h=1}^{n} A_{l h}(s) \int_{s-\eta_{h}(s)}^{s} \widetilde{G}_{h}\left[\varphi_{h}(u)\right] \Delta u+\bigvee_{h=1}^{n} C_{l h}(s) \int_{s-\xi_{\xi_{h}}(s)}^{s} \widetilde{G}_{h}\left[\varphi_{h}(u)\right] \Delta u+\bigwedge_{h=1}^{n} T_{l h}^{*}(s) U_{h}(s)+\bigvee_{h=1}^{n} s_{l h}^{*}(s) U_{h}(s)+I_{l}^{*}(s)\right)\right] \Delta s\right\}=-(\Phi \varphi)_{l}(t), \quad l \in \Lambda, t \in \mathbb{T}$.

From $\left(H_{1}\right)-\left(H_{3}\right)$, we have

$$
\begin{aligned}
\mid & (\Phi \varphi)_{l}^{R}(t)-\left(\varphi^{0}\right)_{l}^{R}(t)|=| \int_{t}^{t+\omega} G_{l}(t, \sigma(s)) \\
& \cdot\left(\sum_{j=1}^{n}\left(b_{l h}^{R}(s) \tilde{f}_{h}^{R}\left[\varphi_{h}\left(s-\tau_{l h}(s)\right)\right]-b_{l h}^{I}(s) \tilde{f}_{h}^{I}\left[\varphi_{h}\left(s-\tau_{l h}(s)\right)\right]-b_{l h}^{J}(s) \tilde{f}_{h}^{J}\left[\varphi_{h}\left(s-\tau_{l h}(s)\right)\right]-b_{l h}^{K}(s) \tilde{f}_{h}^{K}\left[\varphi_{h}\left(s-\tau_{l h}(s)\right)\right]\right)\right. \\
& +\bigwedge_{h=1}^{n} \alpha_{l h}^{R}(s) \int_{s-\eta_{l h}(s)}^{s} \tilde{g}_{h}^{R}\left[\varphi_{h}(u)\right] \Delta u-\bigwedge_{h=1}^{n} \alpha_{l h}^{I}(s) \int_{s-\eta_{l h}(s)}^{s} \tilde{g}_{h}^{I}\left[\varphi_{h}(u)\right] \Delta u-\bigwedge_{h=1}^{n} \alpha_{l h}^{J}(s) \int_{s-\eta_{l h}(s)}^{s} \tilde{g}_{h}^{J}\left[\varphi_{h}(u)\right] \Delta u \\
& -\bigwedge_{h=1}^{n} \alpha_{l h}^{K}(s) \int_{s-\eta_{l h}(s)}^{s} \tilde{g}_{h}^{K}\left[\varphi_{h}(u)\right] \Delta u+\bigvee_{h=1}^{n} \beta_{l h}^{R}(s) \int_{s-\xi_{l h}(s)}^{s} \tilde{g}_{h}^{R}\left[\varphi_{h}(u)\right] \Delta u-\bigvee_{h=1}^{n} \beta_{l h}^{I}(s) \int_{s-\xi_{l h}(s)}^{s} \tilde{g}_{h}^{I}\left[\varphi_{h}(u)\right] \Delta u \\
& \left.-\bigvee_{h=1}^{n} \beta_{l h}^{J}(s) \int_{s-\xi_{l h}(s)}^{s} \tilde{g}_{h}^{J}\left[\varphi_{h}(u)\right] \Delta u-\bigvee_{h=1}^{n} \beta_{l h}^{K}(s) \int_{s-\xi_{l h}(s)}^{s} \tilde{g}_{h}^{K}\left[\varphi_{h}(u)\right] \Delta u\right) \Delta s \mid \leq \int_{t}^{t+\omega} G_{l}(t, \sigma(s)) \\
& \cdot\left(\sum_{h=1}^{n} L_{h}\left(\bar{b}_{l h}^{R}+\bar{b}_{l h}^{I}+\bar{b}_{l h}^{J}+\bar{b}_{l h}^{K}\right)+\sum_{h=1}^{n} \bar{\eta}_{l h} H_{h}\left(\bar{\alpha}_{l h}^{R}+\bar{\alpha}_{l h}^{I}+\bar{\alpha}_{l h}^{I}+\bar{\alpha}_{l h}^{K}\right)+\sum_{h=1}^{n} \bar{\xi}_{l h} H_{h}\left(\bar{\beta}_{l h}^{R}+\bar{\beta}_{l h}^{I}+\bar{\beta}_{l h}^{J}+\bar{\beta}_{l h}^{K}\right)\right) \Delta s\|\varphi\|_{\mathbb{X}} \\
& \leq \frac{\omega \kappa_{l}}{1+\kappa_{l}}\left(\sum_{h=1}^{n} L_{h}\left(\bar{b}_{l h}^{R}+\bar{b}_{l h}^{I}+\bar{b}_{l h}^{J}+\bar{b}_{l h}^{K}\right)+\sum_{h=1}^{n} \bar{\eta}_{l h} H_{h}\left(\bar{\alpha}_{l h}^{R}+\bar{\alpha}_{l h}^{I}+\bar{\alpha}_{l h}^{J}+\bar{\alpha}_{l h}^{K}\right)+\sum_{h=1}^{n} \bar{\xi}_{l h} H_{h}\left(\bar{\beta}_{l h}^{R}+\bar{\beta}_{l h}^{I}+\bar{\beta}_{l h}^{J}+\bar{\beta}_{l h}^{K}\right)\right) \frac{r}{1-\xi} \\
& =\frac{\omega \kappa_{l} P_{l}}{1+\kappa_{l}} \frac{r}{1-\xi} \leq \frac{\xi r}{1-\xi}, \quad l \in \Lambda .
\end{aligned}
$$

In a similar way, one can obtain

$$
\left|(\Phi \varphi)_{l}^{m}(t)-\left(\varphi^{0}\right)_{l}^{m}(t)\right| \leq \frac{\xi r}{1-\xi}
$$

$$
m=I, J, K, l \in \Lambda .
$$

Therefore,

$$
\left\|\Phi \varphi-\varphi^{0}\right\| \leq \frac{\xi r}{1-\xi}
$$

which implies that $\Phi \varphi \in \mathbb{X}^{*}$, so the mapping $\Phi$ is a selfmapping from $\mathbb{X}^{*}$ to $\mathbb{X}^{*}$. Next, we shall prove that $\Phi$ is a contraction mapping. In fact, for any $\varphi, \psi \in \mathbb{X}^{*}$, we can get

$\left|(\Phi \varphi-\Phi \psi)_{l}^{R}(t)\right|=\mid \int_{t}^{t+\omega} G_{l}(t, \sigma(s))$

$\cdot\left(\sum_{j=1}^{n}\left(b_{l h}^{R}(s)\left(\tilde{f}_{h}^{R}\left[\varphi_{h}\left(s-\tau_{l h}(s)\right)\right]-\tilde{f}_{h}^{R}\left[\psi_{h}\left(s-\tau_{l h}(s)\right)\right]\right)-b_{l h}^{I}(s)\left(\tilde{f}_{h}^{I}\left[\varphi_{h}\left(s-\tau_{l h}(s)\right)\right]-\tilde{f}_{h}^{I}\left[\psi_{h}\left(s-\tau_{l h}(s)\right)\right]\right)-b_{l h}^{I}(s)\left(\tilde{f}_{h}^{I}\left[\varphi_{h}\left(s-\tau_{l h}(s)\right)\right]-\tilde{f}_{h}^{J}\left[\psi_{h}\left(s-\tau_{l h}(s)\right)\right]\right)-b_{l h}^{K}(s)\left(\tilde{f}_{h}^{K}\left[\varphi_{h}\left(s-\tau_{l h}(s)\right)\right]-\tilde{f}_{h}^{K}\left[\psi_{h}\left(s-\tau_{l h}(s)\right)\right]\right)\right)\right.$

$+\bigwedge_{h=1}^{n} \alpha_{l h}^{R}(s) \int_{s-\eta_{h}(s)}^{s}\left(\tilde{g}_{h}^{R}\left[\varphi_{h}(u)\right]-\tilde{g}_{h}^{R}\left[\psi_{h}(u)\right]\right) \Delta u-\bigwedge_{h=1}^{n} \alpha_{l h}^{I}(s) \int_{s-\eta_{h k}(s)}^{s}\left(\tilde{g}_{h}^{I}\left[\varphi_{h}(u)\right]-\tilde{g}_{h}^{I}\left[\psi_{h}(u)\right]\right) \Delta u-\bigwedge_{h=1}^{n} \alpha_{l h}^{J}(s) \int_{s-\eta_{h}(s)}^{s}\left(\tilde{g}_{h}^{I}\left[\varphi_{h}(u)\right]-\tilde{g}_{h}^{J}\left[\psi_{h}(u)\right]\right) \Delta u-\bigwedge_{h=1}^{n} \alpha_{l h}^{K}(s) \int_{s-\eta_{\eta_{k}}(s)}^{s}\left(\tilde{g}_{h}^{K}\left[\varphi_{h}(u)\right]-\tilde{g}_{h}^{K}\left[\psi_{h}(u)\right]\right) \Delta u$ $\left.+\bigvee_{h=1}^{n} \beta_{l h}^{R}(s) \int_{s-\xi_{\xi_{h}}(s)}^{s}\left(\tilde{g}_{h}^{R}\left[\varphi_{h}(u)\right]-\tilde{g}_{h}^{R}\left[\psi_{h}(u)\right]\right) \Delta u-\bigvee_{h=1}^{n} \beta_{l h}^{I}(s) \int_{s-\xi_{h}(s)}^{s}\left(\tilde{g}_{h}^{I}\left[\varphi_{h}(u)\right]-\tilde{g}_{h}^{I}\left[\psi_{h}(u)\right]\right) \Delta u-\bigvee_{h=1}^{n} \beta_{l h}^{I}(s) \int_{s-\xi_{\xi_{h}}(s)}^{s}\left(\tilde{g}_{h}^{I}\left[\varphi_{h}(u)\right]-\tilde{g}_{h}^{I}\left[\psi_{h}(u)\right]\right) \Delta u-\bigvee_{h=1}^{n} \beta_{l h}^{K}(s) \int_{s-\xi_{h}(s)}^{s}\left(\tilde{g}_{h}^{K}\left[\varphi_{h}(u)\right]-\tilde{g}_{h}^{K}\left[\psi_{h}(u)\right]\right) \Delta u\right) \Delta s \mid$

$\leq \int_{t}^{t+\omega} G_{l}(t, \sigma(s))\left(\sum_{h=1}^{n} L_{h}\left(\bar{b}_{l h}^{R}+\bar{b}_{l h}^{I}+\overline{\bar{b}}_{l h}^{I}+\bar{b}_{l h}^{k}\right)+\sum_{h=1}^{n} \bar{\eta}_{l h} H_{h}\left(\bar{\alpha}_{l h}^{R}+\bar{\alpha}_{l h}^{I}+\bar{\alpha}_{l h}^{I}+\bar{\alpha}_{l h}^{K}\right)+\sum_{h=1}^{n} \bar{\xi}_{l h} H_{h}\left(\bar{\beta}_{l h}^{R}+\bar{\beta}_{l h}^{I}+\bar{\beta}_{l h}^{I}+\bar{\beta}_{l h}^{K}\right)\right) \Delta s\|\varphi-\psi\|_{x} \leq \frac{\omega \kappa_{l}}{1+\kappa_{l}}\left(\sum_{h=1}^{n} L_{h}\left(\bar{b}_{l h}^{R}+\bar{b}_{l h}^{I}+\bar{b}_{l h}^{I}+\bar{b}_{l h}^{K}\right)+\sum_{h=1}^{n} \overline{\bar{m}}_{l h} H_{h}\left(\bar{\alpha}_{l h}^{R}+\bar{\alpha}_{l h}^{I}+\bar{\alpha}_{l h}^{I}+\bar{\alpha}_{l h}^{K}\right)\right.$

$\left.+\sum_{h=1}^{n} \bar{z}_{l h} H_{h}\left(\bar{\beta}_{l h}^{R}+\bar{\beta}_{l h}^{I}+\bar{\beta}_{l h}^{I}+\bar{\beta}_{l h}^{K}\right)\right)\|\varphi-\psi\|_{X} \leq \xi\|\varphi-\psi\|_{X}, \quad l \in \Lambda$. 
In a similar way, we can obtain

$$
\left|(\Phi \varphi-\Phi \psi)_{l}^{m}(t)\right| \leq \xi\|\varphi-\psi\|_{\mathbb{X}}
$$

$$
m=I, J, K, l \in \Lambda \text {. }
$$

Hence,

$$
\|\Phi \varphi-\Phi \psi\|_{\mathbb{X}} \leq \xi\|\varphi-\psi\|_{\mathbb{X}}
$$

Therefore, by $\left(\mathrm{H}_{3}\right), \Phi$ is a contraction mapping. Thus, system (15) has a unique $\omega$-anti-periodic solution in the region $\mathbb{X}^{*}=$ $\left\{\varphi \in \mathbb{X}:\left\|\varphi-\varphi^{0}\right\|_{\mathbb{X}} \leq \xi r /(1-\xi)\right\}$. This completes the proof of Theorem 14.

\section{Global Exponential Stability of Anti-Periodic Solutions}

In this section, we will study the global exponential stability of anti-periodic solutions of (15).

Theorem 15. Assume that $\left(H_{1}\right)-\left(H_{3}\right)$ hold. Suppose further that

$\left(H_{4}\right) \underline{a}_{l}>\max \left\{P_{l}\right\}, l \in \Lambda$,

then system (15) has an $\omega$-anti-periodic solution that is globally exponentially stable.

Proof. From Theorem 14, we see that system (15) has an $\omega$ anti-periodic solution $Z^{*}(t)=\left(X_{1}^{*}(t), X_{2}^{*}(t), \ldots, X_{n}^{*}(t)\right)^{T}$ with initial value $\varphi^{*}(s)=\left(\varphi_{1}^{*}(t), \varphi_{2}^{*}(t), \ldots, \varphi_{n}^{*}(t)\right)^{T}$. Suppose that $Z(t)=\left(X_{1}(t), X_{2}(t), \ldots, X_{n}(t)\right)^{T}$ is an arbitrary solution of system (15) with initial value $\varphi(s)=\left(\varphi_{1}(t), \varphi_{2}(t)\right.$, $\left.\ldots, \varphi_{n}(t)\right)^{T}$. Then

$$
\begin{aligned}
& \left(Z(t)-Z^{*}(t)\right)_{l}^{\Delta}(t)=-a_{l}(t)\left(X_{l}(t)-X_{l}^{*}(t)\right) \\
& \quad+\sum_{h=1}^{n} B_{l h}(t) \\
& \cdot\left(\widetilde{F}_{h}\left[x_{h}\left(t-\tau_{l h}(t)\right)\right]-\widetilde{F}_{h}\left[x_{h}^{*}\left(t-\tau_{l h}(t)\right)\right]\right) \\
& +\bigwedge_{h=1}^{n} A_{l h}(t) \int_{t-\eta_{l h}(t)}^{t}\left(\widetilde{G}_{h}\left[x_{h}(s)\right]-\widetilde{G}_{h}\left[x_{h}^{*}(s)\right]\right) \Delta s \\
& \quad+\bigvee_{h=1}^{n} C_{l h}(t) \int_{t-\xi_{l h}(t)}^{t}\left(\widetilde{G}_{h}\left[x_{h}(s)\right]-G_{h}\left[x_{h}(s)\right]\right) \Delta s,
\end{aligned}
$$

Let $\Theta_{l}$ be defined by

$$
\begin{aligned}
& \Theta_{l}(\theta)=\underline{a}_{l}-\theta-\exp \left(\theta \sup _{s \in \mathbb{T}} \mu(s)\right) \\
& \cdot\left(\sum_{h=1}^{n} \exp \left(\theta \bar{\tau}_{l h}\right) L_{h}\left(\bar{b}_{l h}^{R}+\bar{b}_{l h}^{I}+\bar{b}_{l h}^{J}+\bar{b}_{l h}^{K}\right)\right. \\
& +\sum_{h=1}^{n} \bar{\eta}_{l h} H_{h}\left(\bar{\alpha}_{l h}^{R}+\bar{\alpha}_{l h}^{I}+\bar{\alpha}_{l h}^{J}+\bar{\alpha}_{l h}^{K}\right) \\
& \left.+\sum_{h=1}^{n} \bar{\xi}_{l h} H_{h}\left(\bar{\beta}_{l h}^{R}+\bar{\beta}_{l h}^{I}+\bar{\beta}_{l h}^{J}+\bar{\beta}_{l h}^{K}\right)\right), \quad l \in \Lambda .
\end{aligned}
$$

By $\left(H_{5}\right)$, for $l \in \Lambda$, we find

$$
\Theta_{l}(0)=\underline{a}_{l}-P_{l}>0 .
$$

Since $\Theta_{l}$ is continuous on $[0,+\infty)$ and $\Theta_{l}(\theta) \longrightarrow-\infty$, as $\theta \longrightarrow+\infty$, so there exists $\theta_{l}$ such that $\Theta_{l}\left(\theta_{l}\right)=0$ and $\Theta_{l}(\theta)>0$ for $\theta \in\left(0, \theta_{l}\right), l \in \Lambda$. By choosing $c=\min _{l \in \Lambda}\left\{\theta_{l}\right\}$, we have $\Theta_{l}(c) \geq 0, l \in \Lambda$. So, we can choose a positive constant $0<\lambda<\min \left\{c, \min _{l \in \Lambda}\left\{\underline{a}_{l}\right\}\right\}$ such that

$$
\Theta_{l}(\lambda)>0, \quad l \in \Lambda,
$$

which imply that

$$
\begin{aligned}
& \frac{\exp \left(\lambda \sup _{s \in \mathbb{T}} \mu(s)\right)}{\underline{a}_{l}-\lambda}\left(\sum_{h=1}^{n} \exp \left(\lambda \bar{\tau}_{l h}\right)\right. \\
& \cdot L_{h}\left(\bar{b}_{l h}^{R}+\bar{b}_{l h}^{I}+\bar{b}_{l h}^{J}+\bar{b}_{l h}^{K}\right)+\sum_{h=1}^{n} \bar{\eta}_{l h} H_{h}\left(\bar{\alpha}_{l h}^{R}+\bar{\alpha}_{l h}^{I}\right. \\
& \left.\left.+\bar{\alpha}_{l h}^{J}+\bar{\alpha}_{l h}^{K}\right)+\sum_{h=1}^{n} \bar{\xi}_{l h} H_{h}\left(\bar{\beta}_{l h}^{R}+\bar{\beta}_{l h}^{I}+\bar{\beta}_{l h}^{J}+\bar{\beta}_{l h}^{K}\right)\right) \\
& <1,
\end{aligned}
$$

where $l \in \Lambda$. Let

$$
M_{*}=\min \left\{\frac{a_{l}}{\overline{P_{l}}}\right\}, \quad l \in \Lambda,
$$

by $\left(H_{4}\right)$, we have $M_{*}>1$. Thus,

$$
\begin{aligned}
& \frac{1}{M_{*}}<\frac{\exp \left(\lambda \sup _{s \in \mathbb{T}} \mu(s)\right)}{\underline{a}_{l}-\lambda}\left(\sum_{h=1}^{n} \exp \left(\lambda \bar{\tau}_{l h}\right)\right. \\
& \cdot L_{h}\left(\bar{b}_{l h}^{R}+\bar{b}_{l h}^{I}+\bar{b}_{l h}^{J}+\bar{b}_{l h}^{K}\right)+\sum_{h=1}^{n} \bar{\eta}_{l h} H_{h}\left(\bar{\alpha}_{l h}^{R}+\bar{\alpha}_{l h}^{I}\right. \\
& \left.\left.+\bar{\alpha}_{l h}^{J}+\bar{\alpha}_{l h}^{K}\right)+\sum_{h=1}^{n} \bar{\xi}_{l h} H_{h}\left(\bar{\beta}_{l h}^{R}+\bar{\beta}_{l h}^{I}+\bar{\beta}_{l h}^{J}+\bar{\beta}_{l h}^{K}\right)\right) .
\end{aligned}
$$

Let $\left\|Z(t)-Z^{*}(t)\right\|=\max _{l \in \Lambda}\left\{\max _{m \in M}\left\{\left|x_{l}^{m}(t)-\left(x_{l}^{*}\right)^{m}(t)\right|\right\}\right\}$, $\|\omega\|_{0}=\max _{l \in \Lambda}\left\{\max _{m \in M}\left\{\sup _{s \in\left[t_{0}-v, t_{0}\right]_{\pi}}\left|\varphi_{l}^{m}(s)-\left(\varphi_{l}^{*}\right)^{m}(s)\right|\right\}\right\}$. For any $\varepsilon>0$, it is obvious that

$$
\left\|Z\left(t_{0}\right)-Z^{*}\left(t_{0}\right)\right\|<\|\bowtie\|_{0}+\varepsilon
$$


and

$$
t \in\left[t_{0}-v, t_{1}\right)_{\mathbb{T}} .
$$

$$
\begin{aligned}
\left\|Z(t)-Z^{*}(t)\right\|<\left(\|\omega\|_{0}+\varepsilon\right) e_{\ominus \lambda}\left(t, t_{0}\right) & \\
<M_{*}\left(\|\omega\|_{0}+\varepsilon\right) e_{\ominus \lambda}\left(t, t_{0}\right) & \\
\forall t & \in\left[t_{0}-\nu, t_{0}\right]_{\mathbb{T}} .
\end{aligned}
$$

We claim that

$$
\begin{aligned}
&\left\|Z(t)-Z^{*}(t)\right\|<M_{*}\left(\|\omega\|_{0}+\varepsilon\right) e_{\ominus \lambda}\left(t, t_{0}\right), \\
& \forall t \in\left(t_{0},+\infty\right)_{\mathbb{T}} .
\end{aligned}
$$

If (47) is not true, then there must be a first time $t_{1} \epsilon$ $\left(t_{0},+\infty\right)_{\mathbb{\mathbb { V }}}$ such that

$$
\begin{array}{r}
\left\|Z\left(t_{1}\right)-Z^{*}\left(t_{1}\right)\right\| \geq M_{*}\left(\|\omega\|_{0}+\varepsilon\right) e_{\ominus \lambda}\left(t_{1}, t_{0}\right), \\
\left\|Z(t)-Z^{*}(t)\right\|<M_{*}\left(\|\omega\|_{0}+\varepsilon\right) e_{\ominus \lambda}\left(t, t_{0}\right), \\
t \in\left[t_{0}-v, t_{1}\right)_{\mathbb{T}} .
\end{array}
$$

Multiplying both sides of (38) by $e_{-d_{l}}\left(t_{0}, \sigma(t)\right)$ and integrating over $\left[t_{0}, t\right]_{\mathbb{T}}$, we get

$$
\left(Z(t)-Z^{*}(t)\right)_{l}=\left(Z\left(t_{0}\right)-Z^{*}\left(t_{0}\right)\right)_{l} e_{-a_{l}}\left(t, t_{0}\right)
$$

$$
\begin{aligned}
& +\int_{t_{0}}^{t} e_{-a_{l}}(t, \sigma(s)) \times\left(\sum_{h=1}^{n} B_{l h}(s)\right. \\
& \cdot\left(\widetilde{F}_{h}\left[x_{h}\left(s-\tau_{l h}(s)\right)\right]-\widetilde{F}_{h}\left[x_{h}^{*}\left(s-\tau_{l h}(s)\right)\right]\right)
\end{aligned}
$$$$
+\bigwedge_{h=1}^{n} A_{l h}(s) \int_{s-\eta_{l h}(s)}^{s}\left(\widetilde{G}_{h}\left[x_{h}(u)\right]-\widetilde{G}_{h}\left[x_{h}^{*}(u)\right]\right) \Delta u
$$

$$
\begin{aligned}
& +\bigvee_{h=1}^{n} C_{l h}(s) \\
& \left.\cdot \int_{s-\xi_{l h}(s)}^{s}\left(\widetilde{G}_{h}\left[x_{h}(u)\right]-\widetilde{G}_{h}\left[x_{h}^{*}(u)\right]\right) \Delta u\right) \Delta s,
\end{aligned}
$$

$t \in \mathbb{T}, l \in \Lambda$.

$$
\begin{aligned}
\left\|Z\left(t_{1}\right)-Z^{*}\left(t_{1}\right)\right\| & =C M_{*}\left(\|\omega\|_{0}+\varepsilon\right) e_{\ominus \lambda}\left(t_{1}, t_{0}\right), \\
\left\|Z(t)-Z^{*}(t)\right\| & <C M_{*}\left(\|\omega\|_{0}+\varepsilon\right) e_{\ominus \lambda}\left(t, t_{0}\right),
\end{aligned}
$$

$\left|x_{l}^{R}\left(t_{1}\right)-\left(x^{*}\right)_{l}^{R}\left(t_{1}\right)\right|=\mid\left(x_{l}^{R}\left(t_{0}\right)-\left(x^{R}\right)_{l}^{*}\left(t_{0}\right)\right) e_{-a_{l}}\left(t_{1}, t_{0}\right)+\int_{t_{0}}^{t_{1}} e_{-a_{l}}\left(t_{1}, \sigma(s)\right)$

$\cdot\left(\sum_{j=1}^{n}\left(b_{l h}^{R}(s)\left(\tilde{f}_{h}^{R}\left[x_{h}\left(s-\tau_{l h}(s)\right)\right]-\tilde{f}_{h}^{R}\left[x_{h}^{*}\left(s-\tau_{l h}(s)\right)\right]\right)-b_{l h}^{I}(s)\left(\tilde{f}_{h}^{I}\left[x_{h}\left(s-\tau_{l h}(s)\right)\right]-\tilde{f}_{h}^{I}\left[x_{h}^{*}\left(s-\tau_{l h}(s)\right)\right]\right)-b_{l h}^{I}(s)\left(\tilde{f}_{h}^{I}\left[x_{h}\left(s-\tau_{l h}(s)\right)\right]-\tilde{f}_{h}^{J}\left[x_{h}^{*}\left(s-\tau_{l h}(s)\right)\right]\right)-b_{l h}^{K}(s)\left(\tilde{f}_{h}^{K}\left[x_{h}\left(s-\tau_{l h}(s)\right)\right]-\tilde{f}_{h}^{K}\left[x_{h}^{*}\left(s-\tau_{l h}(s)\right)\right]\right)\right)\right.$

$+\bigwedge_{h=1}^{n} \alpha_{l h}^{R}(s) \int_{s-\eta_{h}(s)}^{s}\left(\tilde{g}_{h}^{R}\left[x_{h}(u)\right]-\tilde{g}_{h}^{R}\left[x_{h}^{*}(u)\right]\right) \Delta u-\bigwedge_{h=1}^{n} \alpha_{l_{h}^{I}}^{I}(s) \int_{s-\eta_{h}(s)}^{s}\left(\tilde{g}_{h}^{I}\left[x_{h}(u)\right]-\tilde{g}_{h}^{I}\left[x_{h}^{*}(u)\right]\right) \Delta u-\bigwedge_{h=1}^{n} \alpha_{h h}^{J}(s) \int_{s-\eta_{h}(s)}^{s}\left(\tilde{g}_{h}^{I}\left[x_{h}(u)\right]-\tilde{g}_{h}^{I}\left[x_{h}^{*}(u)\right]\right) \Delta u-\bigwedge_{h=1}^{n} \alpha_{l h}^{K}(s) \int_{s-\eta_{h}(s)}^{s}\left(\tilde{g}_{h}^{K}\left[x_{h}(u)\right]-\tilde{g}_{h}^{K}\left[x_{h}^{*}(u)\right]\right) \Delta u$

$\left.+\bigvee_{h=1}^{n} \beta_{l h}^{R}(s) \int_{s-\xi_{h}(s)}^{s}\left(\tilde{g}_{h}^{R}\left[x_{h}(u)\right]-\tilde{g}_{h}^{R}\left[x_{h}^{*}(u)\right]\right) \Delta u-\bigvee_{h=1}^{n} \beta_{l h}^{I}(s) \int_{s-\xi_{\xi_{h}(s)}(s)}^{s}\left(\tilde{g}_{h}^{I}\left[x_{h}(u)\right]-\tilde{g}_{h}^{I}\left[x_{h}^{*}(u)\right]\right) \Delta u-\bigvee_{h=1}^{n} \beta_{l h}^{I}(s) \int_{s-\xi_{\xi_{h}}(s)}^{s}\left(\tilde{g}_{h}^{I}\left[x_{h}(u)\right]-\tilde{g}_{h}^{I}\left[x_{h}^{*}(u)\right]\right) \Delta u-\bigvee_{h=1}^{n} \beta_{l h}^{K}(s) \int_{s-\xi_{h}(s)}^{s}\left(\tilde{g}_{h}^{K}\left[x_{h}(u)\right]-\tilde{g}_{h}^{K}\left[x_{h}^{*}(u)\right]\right) \Delta u\right) \Delta s \mid$

$\leq\left\{\frac{e_{-a_{q} \oplus \lambda}\left(t_{1}, t_{0}\right)}{C M_{s}}+\int_{t_{0}}^{t_{1}} e_{-a, \oplus \lambda}\left(t_{1}, \sigma(s)\right)\left[e_{\lambda}\left(\sigma(s), s-\tau_{l h}(s)\right) \sum_{h=1}^{n} L_{h}\left(\bar{b}_{l h}^{R}+\bar{b}_{l h}^{I}+\bar{b}_{l h}^{I}+\bar{b}_{l h}^{K}\right)+e_{\lambda}(\sigma(s), s) \sum_{h=1}^{n} \bar{\eta}_{l h} H_{h}\left(\bar{\alpha}_{l h}^{R}+\bar{\alpha}_{l h}^{I}+\bar{\alpha}_{l h}^{I}+\bar{\alpha}_{l h}^{K}\right)+e_{\lambda}(\sigma(s), s) \times \sum_{h=1}^{n} \bar{\xi}_{l h} H_{h}\left(\bar{\beta}_{l h}^{R}+\bar{\beta}_{l h}^{I}+\bar{\beta}_{l h}^{I}+\bar{\beta}_{l h}^{K}\right)\right] \Delta s\right\} C M_{s}\left(\|\omega\|_{0}+\varepsilon\right) e_{\ominus \lambda}\left(t_{1}, t_{0}\right)$

$\leq\left\{\frac{1}{M_{*}}-\frac{\exp \left(\lambda \sup _{s \in \top} \mu(s)\right)}{\underline{a}_{l}-\lambda}\left[\exp \left(\lambda \bar{\tau}_{l h}\right) \sum_{h=1}^{n} L_{h}\left(\bar{b}_{l h}^{R}+\bar{b}_{l h}^{I}+\bar{b}_{l h}^{I}+\bar{b}_{l h}^{K}\right)+\sum_{h=1}^{n} \bar{\eta}_{l h} H_{h}\left(\bar{\alpha}_{l h}^{R}+\bar{\alpha}_{l h}^{I}+\bar{\alpha}_{l h}^{I}+\bar{\alpha}_{l h}^{K}\right)+\sum_{h=1}^{n} \bar{\xi}_{l h} H_{h}\left(\bar{\beta}_{l h}^{R}+\bar{\beta}_{l h}^{I}+\bar{\beta}_{l h}^{I}+\bar{\beta}_{l h}^{K}\right)\right] e_{-\left(a_{l}-\lambda\right)}\left(t_{1}, t_{0}\right)+\frac{\exp \left(\lambda \sup _{s \in \in} \mu(s)\right)}{\underline{a}_{l}-\lambda}\left[\exp \left(\lambda \bar{\tau}_{l h}\right) \sum_{h=1}^{n} L_{h}\left(\bar{b}_{l h}^{R}+\bar{b}_{l h}^{I}+\bar{b}_{l h}^{I}+\bar{b}_{l h}^{K}\right)\right.\right.$

$\left.\left.+\sum_{h=1}^{n} \bar{\eta}_{l h} H_{h}\left(\bar{\alpha}_{l h}^{R}+\bar{\alpha}_{l h}^{I}+\bar{\alpha}_{l h}^{I}+\bar{\alpha}_{l h}^{K}\right)+\sum_{h=1}^{n} \bar{\xi}_{l h} H_{h}\left(\bar{\beta}_{l h}^{R}+\bar{\beta}_{l h}^{I}+\bar{\beta}_{l h}^{I}+\bar{\beta}_{l h}^{K}\right)\right]\right\} C M_{*}\left(\|\oplus\|_{0}+\varepsilon\right) e_{\ominus \lambda}\left(t_{1}, t_{0}\right)<C M_{*}\left(\|\oplus\|_{0}+\varepsilon\right) e_{\ominus \lambda}\left(t_{1}, t_{0}\right), \quad l \in \Lambda$.

Similarly, we have

$$
\begin{array}{r}
\left|x_{l}^{m}\left(t_{1}\right)-\left(x^{*}\right)_{l}^{m}\left(t_{1}\right)\right|<C M_{*}\left(\|\omega\|_{0}+\varepsilon\right) e_{\ominus \lambda}\left(t_{1}, t_{0}\right), \\
m=I, J, K, l \in \Lambda .
\end{array}
$$

Hence

$$
\begin{aligned}
& \| Z\left(t_{1}\right)-Z^{*}\left(t_{1}\right) \| \\
& \quad=\max \left\{\left|x_{l}^{m}\left(t_{1}\right)-\left(x^{*}\right)_{l}^{m}\left(t_{1}\right)\right|, m \in M, l \in \Lambda\right\} \\
& \quad<C M_{*}\left(\|\omega\|_{0}+\varepsilon\right) e_{\ominus \lambda}\left(t_{1}, t_{0}\right),
\end{aligned}
$$

which contradicts the first equation of (48). Therefore, (47) holds. Letting $\varepsilon \longrightarrow 0^{+}$leads to

$$
\begin{aligned}
&\left\|Z(t)-Z^{*}(t)\right\| \leq M_{*}\|\omega\|_{0} e_{\ominus \lambda}\left(t, t_{0}\right) \\
& \forall t \in\left(t_{0},+\infty\right)_{\mathbb{T}} .
\end{aligned}
$$

Hence, the $\omega$-anti-periodic solution of system (15) is globally exponentially stable. The proof of Theorem 15 is completed.

Remark 16. In view of Remark 11, we have that, under conditions of Theorems 14 and 15, system (10) has a unique anti-periodic solution that is globally exponentially stable. 
Remark 17. In view of Theorem 14, Theorem 15, and Remark 16, we see that under condition $-a_{l} \in \mathscr{R}^{+}, l \in \Lambda$ both the continuous time and the discrete time cases of system (10) have the same dynamics for the anti-periodicity.

\section{An Example}

In this section, we give an example to illustrate the feasibility and effectiveness of our results obtained in Sections 3 and 4.

Example 18. In system (10), let $n=2, \omega=\pi$. The coefficients are taken as follows:

$$
\begin{aligned}
& \tilde{f}_{h}(x)=\tilde{g}_{h}(x) \\
& =\frac{1}{80 h}\left(\left|x^{R}+1\right|-\left|x^{J}-1\right|\right) \\
& +i \frac{h}{80} \sin \frac{\sqrt{2}}{2}\left(x^{I}+x^{K}\right)-j \frac{h}{80} \sin x^{J} \\
& +\frac{h}{80} k \tanh \left(x^{I}+x^{K}+x^{R}\right), \\
& a_{1}(t)=0.8+0.02|\cos t|, \\
& a_{2}(t)=0.95+0.01|\cos t| \text {, } \\
& I_{l}(t)=0.2 l \cos t+i(0.1+0.2 \sin t)+0.2 j \sin t \\
& +0.1 k \cos t \\
& b_{l h}(t)=0.4 \sin 2 t+0.1 h i \cos 2 t+0.1 l j \cos 2 t \\
& +0.3 k \sin 2 t \\
& d_{l h}(t)=0.1 l \cos 2 t+0.3 i \sin 2 t+0.1 h j \cos 2 t \\
& +0.1 k \sin 2 t, \\
& \alpha_{l h}(t)=0.1 h \cos 2 t+0.2 h i \sin 2 t+0.2 j \sin 2 t \\
& +0.1 k \sin 2 t, \\
& \beta_{l h}(t)=0.3 \sin 2 t+0.1 i \cos 2 t+0.2 h j \cos 2 t \\
& +0.2 k \sin 2 t \\
& T_{l h}(t)=0.2+0.1 h \sin 2 t+0.1 i \cos 2 t+0.1 l j \sin 2 t \\
& +0.1 l k \cos 2 t \text {, } \\
& S_{l h}(t)=0.1 \cos 2 t+0.1 h i \sin 2 t+0.1 j \cos 2 t \\
& +0.2 l k \sin 2 t, \\
& \mu_{h}(t)=0.1 l \sin t+0.2 h i \cos t+0.2 j \cos t \\
& +0.1 k \sin t
\end{aligned}
$$

if $\mathbb{T}=\mathbb{R}$, then we take

$$
\begin{aligned}
& \tau_{l h}(t)=\cos ^{2} t, \\
& \eta_{l h}(t)=\frac{h}{2}|\cos t|, \\
& \xi_{l h}(t)=\frac{l}{2} \sin ^{2} t,
\end{aligned}
$$

and if $\mathbb{U}=\mathbb{Z}$, then we take

$$
\begin{aligned}
\tau_{l h}(t) & =1+\cos ^{2}\left(\frac{\pi}{2}+\pi t\right) \\
\eta_{l h}(t) & =\frac{h}{2}|\sin \pi t| \\
\xi_{l h}(t) & =\frac{l}{2} \sin ^{2} \pi t
\end{aligned}
$$

$$
l, h=1,2 .
$$

By calculating, we have

$$
\begin{aligned}
& L_{1}^{m}=H_{1}^{m}=0.0125 \text {, } \\
& L_{2}^{m}=H_{2}^{m}=0.025 \text {, } \\
& \bar{a}_{1}=0.82 \text {, } \\
& \bar{a}_{2}=0.96 \text {, } \\
& \underline{a}_{1}=0.8, \\
& \underline{a}_{2}=0.95 \text {, } \\
& \bar{b}_{11}^{R}=0.4 \text {, } \\
& \bar{b}_{11}^{I}=\bar{b}_{11}^{J}=\bar{b}_{12}^{J}=0.1 \text {, } \\
& \bar{b}_{11}^{K}=0.3 \text {, } \\
& \bar{b}_{12}^{R}=\bar{b}_{21}^{R}=0.4 \text {, } \\
& \bar{b}_{12}^{I}=0.2 \text {, } \\
& \bar{b}_{12}^{K}=0.3 \text {, } \\
& \bar{b}_{21}^{I}=0.1 \text {, } \\
& \bar{b}_{21}^{J}=0.2 \text {, } \\
& \bar{b}_{21}^{K}=0.3 \text {, } \\
& \bar{b}_{22}^{R}=0.4 \text {, } \\
& \bar{b}_{22}^{I}=\bar{b}_{22}^{J}=0.2 \text {, } \\
& \bar{b}_{22}^{K}=\bar{d}_{11}^{I}=0.3 \text {, } \\
& \bar{d}_{11}^{R}=\bar{d}_{11}^{J}=\bar{d}_{11}^{K}=0.1, \\
& \bar{d}_{12}^{R}=\bar{d}_{12}^{K}=0.1 \text {, } \\
& \bar{d}_{12}^{I}=0.3 \text {, } \\
& \bar{d}_{12}^{J}=\bar{d}_{21}^{R}=0.2 \text {, }
\end{aligned}
$$



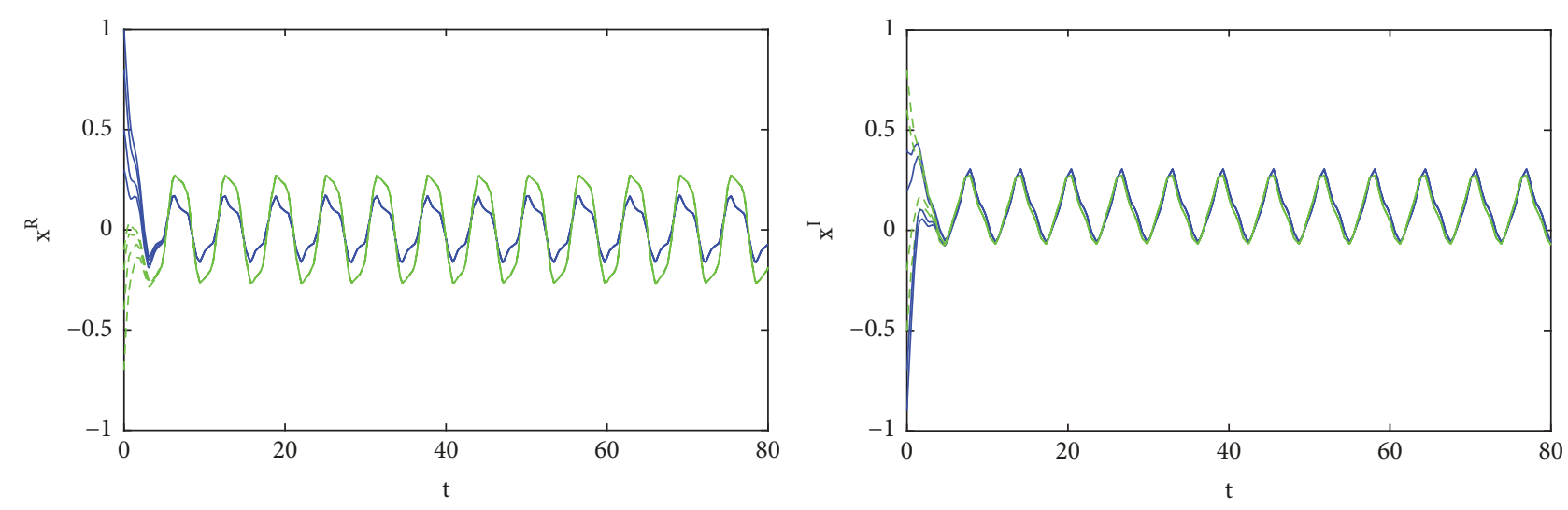

\section{$-\mathrm{x}_{1}^{\mathrm{R}}$ \\ $---x_{2}^{R}$}

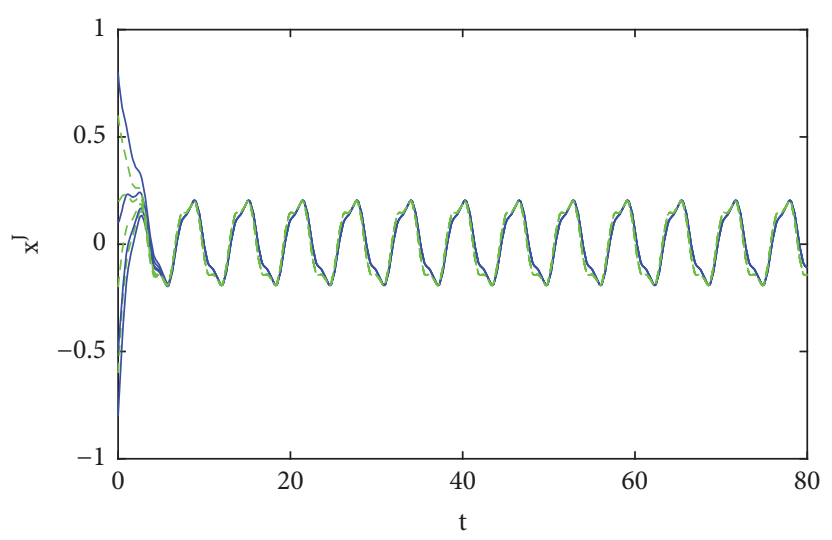

$\begin{aligned} &-x_{1}^{J} \\ &--- x_{2}^{J}\end{aligned}$
$-\mathrm{x}_{1}^{\mathrm{I}}$

$--x_{2}^{I}$

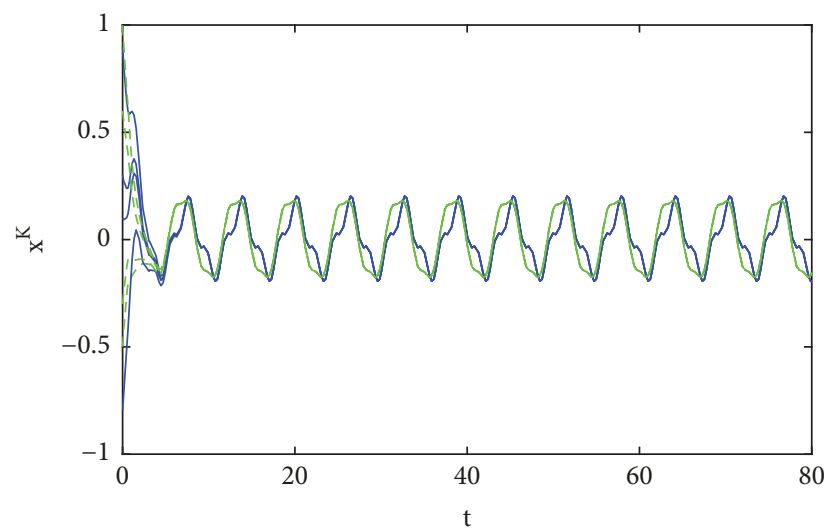

$-\mathrm{x}_{1}^{\mathrm{K}}$

FIGURE 1: Responses of the four parts of $x$ with continuous time t.

$\bar{d}_{21}^{I}=0.1$

$\bar{d}_{21}^{J}=\bar{d}_{21}^{K}=0.1$,

$\bar{d}_{22}^{R}=\bar{d}_{22}^{J}=0.2$,

$\bar{d}_{22}^{I}=0.3$,

$\bar{d}_{22}^{K}=0.1$,

$\bar{\tau}_{11}=\bar{\tau}_{12}=\bar{\tau}_{21}=\bar{\tau}_{22}=1$,

$\bar{\alpha}_{11}^{R}=\bar{\alpha}_{11}^{K}=0.1$,

$\bar{\alpha}_{11}^{J}=\bar{\alpha}_{11}^{I}=\bar{\alpha}_{12}^{R}=\bar{\alpha}_{12}^{J}=0.2$,

$\bar{\alpha}_{12}^{I}=0.4$,

$\bar{\alpha}_{12}^{K}=0.1$,

$\bar{\alpha}_{21}^{R}=0.1$,

$\bar{\alpha}_{21}^{I}=\bar{\alpha}_{21}^{J}=\bar{\alpha}_{22}^{J}=\bar{\alpha}_{22}^{R}=0.2$,

$$
\begin{aligned}
& \bar{\alpha}_{21}^{K}=\bar{\alpha}_{22}^{K}=0.1, \\
& \bar{\alpha}_{22}^{I}=0.4, \\
& \bar{\beta}_{11}^{R}=\bar{\beta}_{21}^{R}=\bar{\beta}_{12}^{R}=\bar{\beta}_{22}^{R}=0.3, \\
& \bar{\beta}_{11}^{I}=\bar{\beta}_{12}^{I}=\bar{\beta}_{21}^{I}=\bar{\beta}_{22}^{I}=0.1, \\
& \bar{\beta}_{12}^{J}=\bar{\beta}_{22}^{J}=0.4, \\
& \bar{\beta}_{11}^{J}=\bar{\beta}_{21}^{J}=0.2, \\
& \bar{\beta}_{12}^{K}=\bar{\beta}_{11}^{K}=\bar{\beta}_{21}^{K}=\bar{\beta}_{22}^{K}=0.2,
\end{aligned}
$$

when $\mathbb{T}=\mathbb{R}$,

$$
\begin{aligned}
& \bar{\xi}_{11}=\bar{\xi}_{12}=0.5 \\
& \bar{\xi}_{21}=\bar{\xi}_{22}=1
\end{aligned}
$$



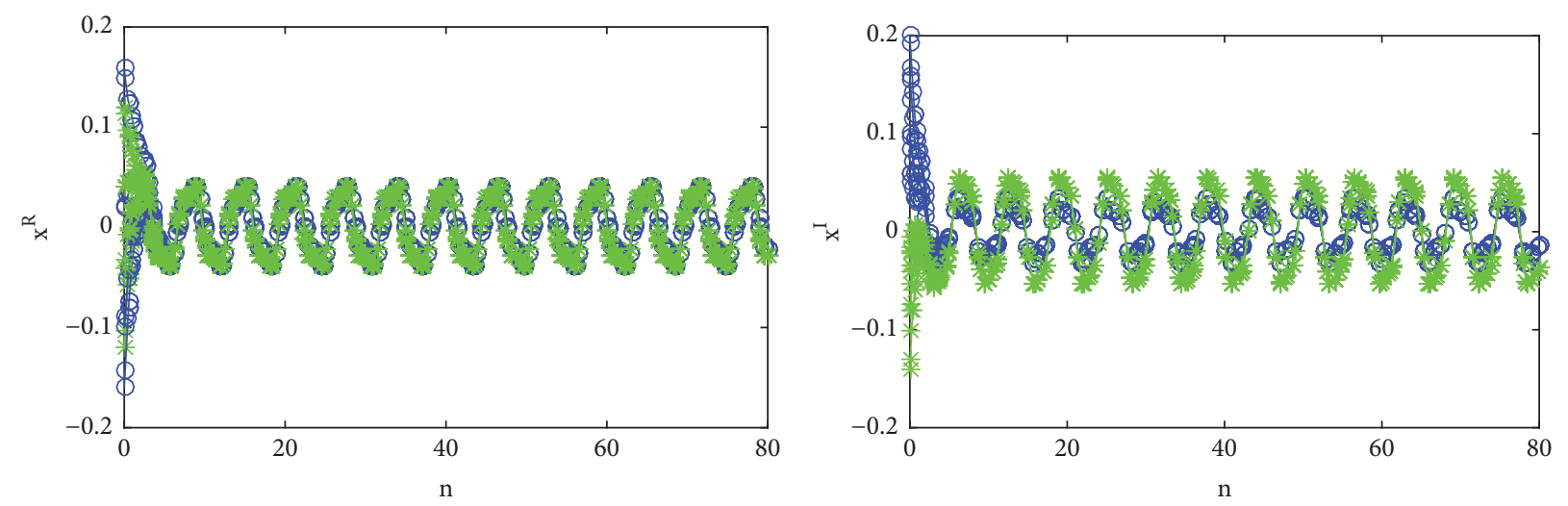

$\ominus \mathrm{x}_{1}^{\mathrm{R}}$
$-\mathrm{x}_{2}^{\mathrm{R}}$

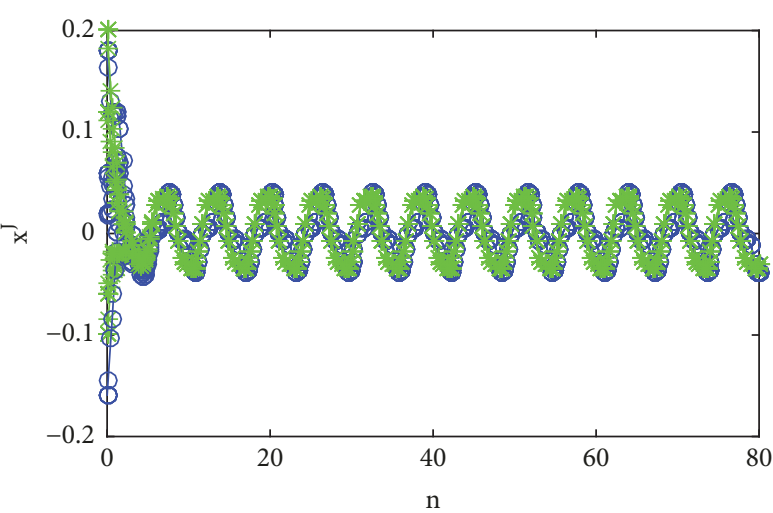

$\ominus x_{1}^{J}$
$-x_{2}^{J}$ $\ominus \mathrm{x}_{1}^{\mathrm{I}}$

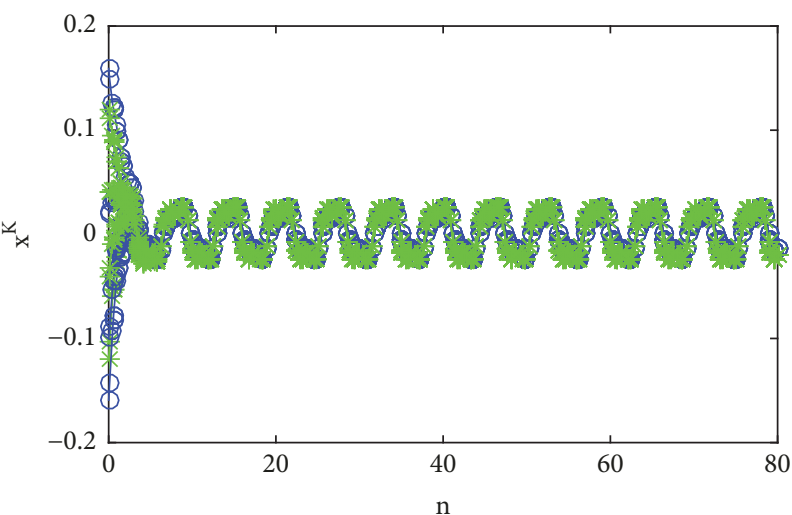

$\circ x_{1}^{K}$
$-* x_{2}^{K}$

FIgURE 2: Responses of the four parts of $x$ with discrete time t.

$$
\begin{aligned}
& \bar{\eta}_{11}=\bar{\eta}_{21}=0.5, \\
& \bar{\eta}_{12}=\bar{\eta}_{22}=1,
\end{aligned}
$$

when $\mathbb{T}=\mathbb{Z}$,

$$
\bar{\xi}_{11}=\bar{\xi}_{12}=\bar{\xi}_{21}=\bar{\xi}_{22}=\bar{\eta}_{11}=\bar{\eta}_{21}=\bar{\eta}_{12}=\bar{\eta}_{22}=0 .
$$

Therefore, whether $\mathbb{T}=\mathbb{R}$ or $\mathbb{T}=\mathbb{Z}$, we have $-a_{l} \in \mathscr{R}^{+}$and it is easy to verify that conditions $\left(H_{1}\right)$ and $\left(H_{2}\right)$ are satisfied. By a simple calculation, we have

$$
\xi \leq \max \left\{\frac{P_{1}}{\underline{a}_{1}}, \frac{P_{2}}{\underline{a}_{2}}\right\}=\max \{0.4,0.5\}=0.5<1,
$$

which implies that conditions $\left(\mathrm{H}_{3}\right)$ and $\left(\mathrm{H}_{4}\right)$ are also satisfied. Therefore, according to Theorems 14 and 15, (10) has a unique $\pi$-anti-periodic solution, which is globally exponentially stable (Figures 1 and 2).

\section{Conclusion}

In this paper, we have proposed a class of QVFCNNs with time-varying delays on time scales which can unify the continuous time and discrete time cases of QVFCNNs and contain real-valued FCNNs and complex-valued FCNNs as their special cases. Based on a novel method that is different from those used in $[10,25-34]$, we established the existence and global exponential stability of anti-periodic solutions of the QVFCNNs. To the best of our knowledge, this is the first paper to study the existence of anti-periodic solutions for QVFCNNs with time-varying delays on time scales. The method of this paper may be applied to study other types of the QVNNs on times scales such as quaternionvalued recurrent neural networks, quaternion-valued BAM neural networks, quaternion-valued shunting inhibitory cellular neural networks, quaternion-valued Cohen-Grossberg neural networks, and so on.

\section{Data Availability}

No data were used to support this study.

\section{Conflicts of Interest}

The authors declare that they have no conflicts of interest. 


\section{Acknowledgments}

This work is supported by the National Natural Sciences Foundation of People's Republic of China under Grant 11361072 and the Scientific Research Fund Project of the Yunnan Provincial Education Department of People's Republic of China under Grant 2018JS307.

\section{References}

[1] T. Yang, L.-B. Yang, C. W. Wu, and L. O. Chua, "Fuzzy cellular neural networks: theory," in Proceedings of the 1996 4th IEEE International Workshop on Cellular Neural Networks, and Their Applications, CNNA-96, pp. 181-186, June 1996.

[2] T. Yang, L.-B. Yang, C. W. Wu, and L. O. Chua, "Fuzzy cellular neural networks: applications," in Proceedings of the 1996 4th IEEE International Workshop on Cellular Neural Networks, and Their Applications, CNNA-96, pp. 225-230, June 1996.

[3] G. Yang, Y. Kao, W. Li, and S. Xiqian, "Exponential stability of impulsive stochastic fuzzy cellular neural networks with mixed delays and reaction-diffusion terms," Neural Computing and Applications, vol. 23, no. 3-4, pp. 1109-1121, 2013.

[4] L. Chen and R. Wu, "Exponential stability of stochastic fuzzy cellular neural networks with distributed delays," International Journal of Bifurcation and Chaos, vol. 19, no. 10, pp. 3387-3395, 2009.

[5] P. Balasubramaniam and V. Vembarasan, "Robust stability of uncertain fuzzy BAM neural networks of neutral-type with Markovian jumping parameters and impulses," Computers \& Mathematics with Applications. An International Journal, vol. 62, no. 4, pp. 1838-1861, 2011.

[6] Q. Zhang and R. Xiang, "Global asymptotic stability of fuzzy cellular neural networks with time-varying delays," Physics Letters A, vol. 372, no. 22, pp. 3971-3977, 2008.

[7] S. Long and L. Jia, "Stability analysis of neutral-type fuzzy neural networks with distributed delays," in Proceedings of the 2011 7th International Conference on Computational Intelligence and Security, CIS 2011, pp. 407-411, December 2011.

[8] T. Yang and L.-B. Yang, "The global stability of fuzzy cellular neural network," IEEE Transactions on Circuits and Systems I: Fundamental Theory and Applications, vol. 43, no. 10, pp. 880$883,1996$.

[9] K. Yuan, J. Cao, and J. Deng, "Exponential stability and periodic solutions of fuzzy cellular neural networks with time-varying delays," Neurocomputing, vol. 69, no. 13-15, pp. 1619-1627, 2006.

[10] B. Li and Y. Li, "Anti-periodic solutions for neutral type FCNNs with time-varying delays and D operator on time scales," Journal of Nonlinear Sciences and Applications. JNSA, vol. 10, no. 11, pp. 6119-6131, 2017.

[11] Z. D. Huang, "Almost periodic solutions for fuzzy cellular neural networks with multi-proportional delays," International Journal of Machine Learning and Cybernetics, vol. 8, no. 4, pp. 1323-1331, 2017.

[12] L. Luo, H. Feng, and L. Ding, "Color image compression based on quaternion neural network principal component analysis," in Proceedings of the 2010 International Conference on Multimedia Technology, ICMT 2010, China, October 2010.

[13] N. Matsui, T. Isokawa, H. Kusamichi, F. Peper, and H. Nishimura, "Quaternion neural network with geometrical operators," Journal of Intelligent \& Fuzzy Systems: Applications in Engineering and Technology, vol. 15, no. 3-4, pp. 149-164, 2004.
[14] M. Yoshida, Y. Kuroe, and T. Mori, "Models of hopfield-type quaternion neural networks and their energy functions," International Journal of Neural Systems, vol. 15, no. 1-2, pp. 129-135, 2005.

[15] Y. Liu, D. Zhang, and J. Lu, "Global exponential stability for quaternion-valued recurrent neural networks with time-varying delays," Nonlinear Dynamics, vol. 87, no. 1, pp. 553-565, 2017.

[16] Y. Liu, D. Zhang, J. Lu, and J. Cao, "Global $\mu$-stability criteria for quaternion-valued neural networks with unbounded timevarying delays," Information Sciences, vol. 360, pp. 273-288, 2016.

[17] X. Chen, Z. Li, Q. Song, J. Hu, and Y. Tan, "Robust stability analysis of quaternion-valued neural networks with time delays and parameter uncertainties," Neural Networks, vol. 91, pp. 5565, 2017.

[18] M. E. Valle and F. Z. de Castro, "On the dynamics of Hopfield neural networks on unit quaternions," IEEE Transactions on Neural Networks and Learning Systems, vol. 29, pp. 2464-2471, 2018.

[19] X. F. Chen, Q. K. Song, Z. S. Li, Z. J. Zhao, and Y. R. Liu, “Stability analysis of continuous-time and discrete-time quaternionvalued neural networks with linear threshold neurons," IEEE Transactions on Neural Networks and Learning Systems, vol. 29, pp. 2769-2781, 2018.

[20] Z. Tu, J. Cao, A. Alsaedi, and T. Hayat, "Global dissipativity analysis for delayed quaternion-valued neural networks," Neural Networks, vol. 89, pp. 97-104, 2017.

[21] Y. Li and J. Qin, "Existence and global exponential stability of periodic solutions for quaternion-valued cellular neural networks with time-varying delays," Neurocomputing, vol. 292, pp. 91-103, 2018.

[22] Y. Li and X. Meng, "Existence and global exponential stability of pseudo almost periodic solutions for neutral type quaternionvalued neural networks with delays in the leakage term on time scales," Complexity, Article ID 9878369, 15 pages, 2017.

[23] Y. Li, X. Meng, and Y. Ye, "Almost periodic synchronization for quaternion-valued neural networks with time-varying delays," Complexity, vol. 2018, 13 pages, 2018.

[24] Y. Li, B. Li, S. Yao, and L. Xiong, "The global exponential pseudo almost periodic synchronization of quaternion-valued cellular neural networks with time-varying delays," Neurocomputing, vol. 303, pp. 75-87, 2018.

[25] J. Y. Shao, "Anti-periodic solutions for shunting inhibitory cellular neural networks with time-varying delays," Physics Letters A, vol. 372, no. 30, pp. 5011-5016, 2008.

[26] X. Liu and J. Cao, "Exponential stability of anti-periodic solutions for neural networks with multiple discrete and distributed delays," Proceedings of the Institution of Mechanical Engineers, Part I: Journal of Systems and Control Engineering, vol. 223, no. 3, pp. 299-308, 2008.

[27] A. Abdurahman and H. Jiang, "The existence and stability of the anti-periodic solution for delayed CohenCGrossberg neural networks with impulsive effects," Neurocomputing, vol. 149, pp. 22-28, 2015.

[28] Y. Li and J. Shu, "Anti-periodic solutions to impulsive shunting inhibitory cellular neural networks with distributed delays on time scales," Communications in Nonlinear Science and Numerical Simulation, vol. 16, no. 8, pp. 3326-3336, 2011.

[29] L. Peng and W. Wang, "Anti-periodic solutions for shunting inhibitory cellular neural networks with time-varying delays in leakage terms," Neurocomputing, vol. 111, pp. 27-33, 2013. 
[30] X. Wei and Z. Qiu, "Anti-periodic solutions for BAM neural networks with time delays," Applied Mathematics and Computation, vol. 221, pp. 221-229, 2013.

[31] Q. Zhou, "Anti-periodic solutions for cellular neural networks with oscillating coefficients in leakage terms," International Journal of Machine Learning and Cybernetics, vol. 8, pp. 16071613, 2017.

[32] Z. Chen, "Global exponential stability of anti-periodic solutions for neutral type CNNs with D operator," International Journal of Machine Learning and Cybernetics, vol. 9, pp. 1109-1115, 2018.

[33] C. Xu and P. Li, "On anti-periodic solutions for neutral shunting inhibitory cellular neural networks with time-varying delays and D operator," Neurocomputing, vol. 275, pp. 377-382, 2018.

[34] Q. Zhang, F. Lin, and X. Zhong, "Existence and globally exponential stability of anti periodic solution for fuzzy BAM neural networks with time delays," Applied Mathematics and Computation, vol. 57, no. 1-2, pp. 729-743, 2018.

[35] S. Hilger, "Analysis on measure chains: a unified approach to continuous and discrete calculus," Results in Mathematics, vol. 18, no. 1-2, pp. 18-56, 1990.

[36] M. Bohner and A. Peterson, Dynamic Equations on Time Scales: An Introduction with Applications, Birkhauser, Boston, Mass, USA, 2001.

[37] Y. Li and T. Zhang, "Global exponential stability of fuzzy interval delayed neural networks with impulses on time scales," International Journal of Neural Systems, vol. 19, no. 6, pp. 449-456, 2009.

[38] Y. Li, X. Chen, and L. Zhao, "Stability and existence of periodic solutions to delayed Cohen-Grossberg BAM neural networks with impulses on time scales," Neurocomputing, vol. 72, no. 7-9, pp. 1621-1630, 2009.

[39] M. Syed Ali and J. Yogambigai, "Synchronization of complex dynamical networks with hybrid coupling delays on time scales by handling multitude Kronecker product terms," Applied Mathematics and Computation, vol. 291, pp. 244-258, 2016.

[40] W. Yang, W. Yu, J. Cao, F. E. Alsaadi, and T. Hayat, "Almost automorphic solution for neutral type high-order Hopfield BAM neural networks with time-varying leakage delays on time scales," Neurocomputing, vol. 267, pp. 241-260, 2017.

[41] Y. Li, X. Meng, and L. Xiong, "Pseudo almost periodic solutions for neutral type high-order Hopfield neural networks with mixed time-varying delays and leakage delays on time scales," International Journal of Machine Learning and Cybernetics, vol. 8, no. 6, pp. 1915-1927, 2017.

[42] Y. Li and L. Zhao, "Weighted pseudo-almost periodic functions on time scales with applications to cellular neural networks with discrete delays," Mathematical Methods in the Applied Sciences, vol. 40, no. 6, pp. 1905-1921, 2017.

[43] Q. Wang and Z. Liu, "Existence and stability of positive almost periodic solutions for a competitive system on time scales," Mathematics and Computers in Simulation, vol. 138, pp. 65-77, 2017.

[44] Z. Zhang, D. Hao, and D. Zhou, "Global asymptotic stability by complex-valued inequalities for complex-valued neural networks with delays on period time scales," Neurocomputing, vol. 219, pp. 494-501, 2017.

[45] A. Sudbery, "Quaternionic analysis," Mathematical Proceedings of the Cambridge Philosophical Society, vol. 85, no. 2, pp. 199$224,1979$.

[46] E. R. Kaufmann and Y. N. Raffoul, "Periodic solutions for a neutral nonlinear dynamical equation on a time scale," Journal of Mathematical Analysis and Applications, vol. 319, no. 1, pp. 315-325, 2006.

[47] L. Bi, M. Bohner, and M. Fan, "Periodic solutions of functional dynamic equations with infinite delay," Nonlinear Analysis. Theory, Methods \& Applications. An International Multidisciplinary Journal, vol. 68, no. 5, pp. 1226-1245, 2008. 


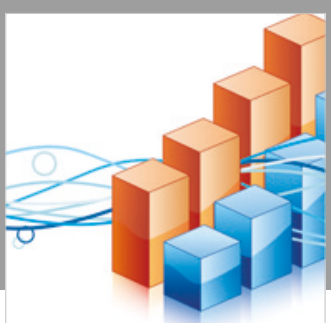

Advances in

Operations Research

\section{-n-m}
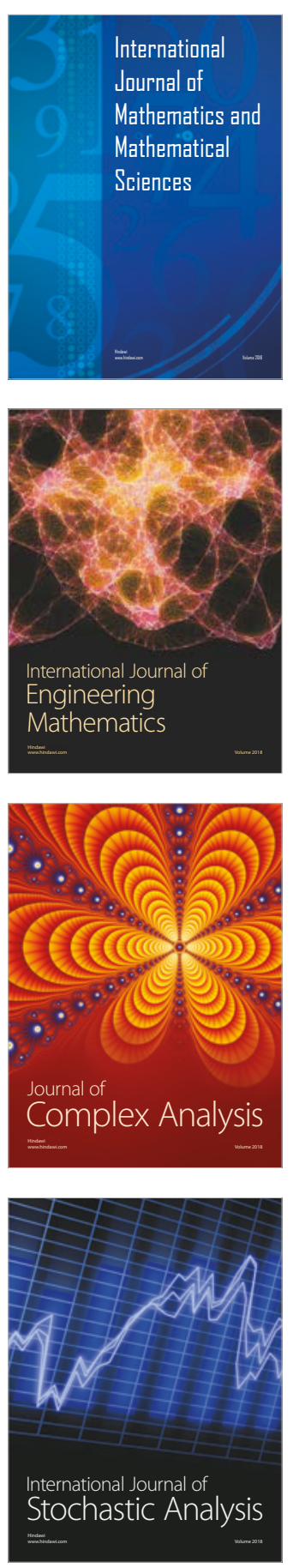
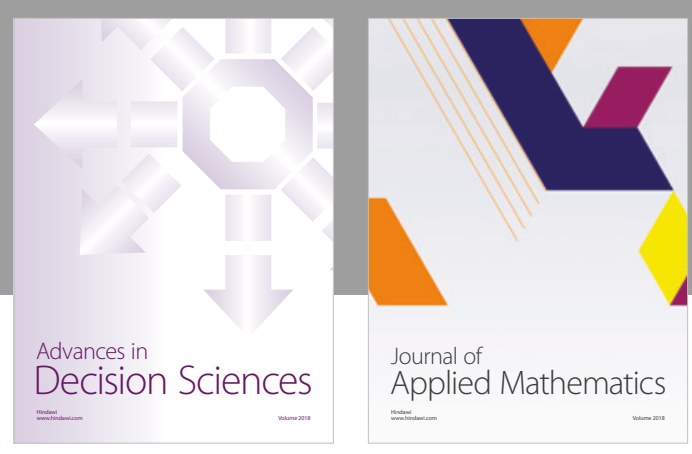

Journal of

Applied Mathematics
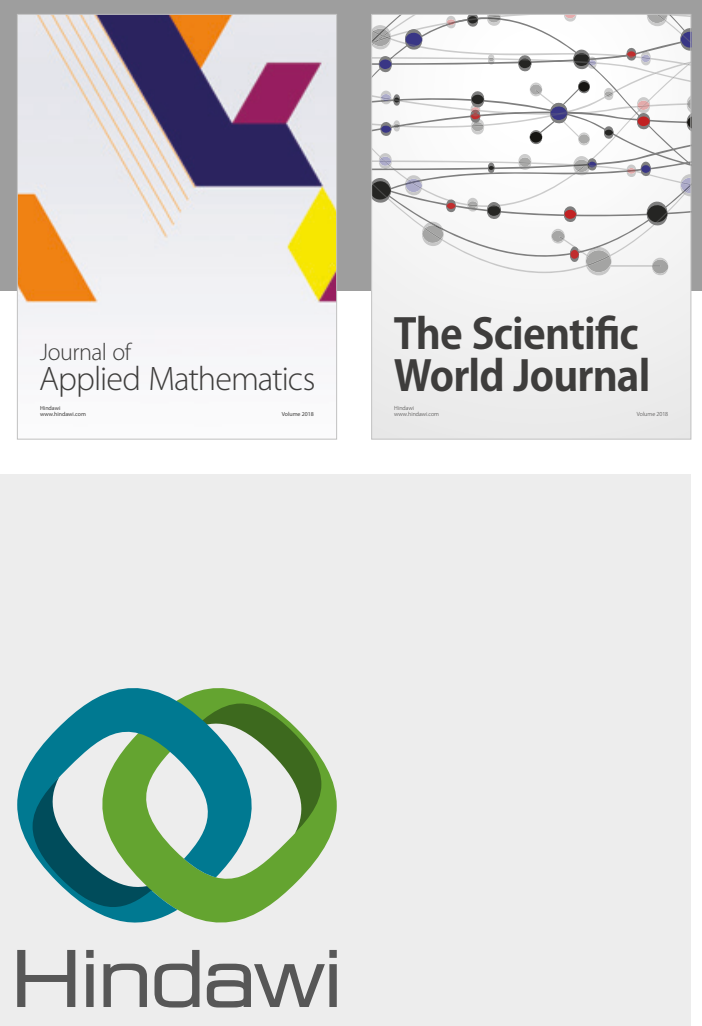

Submit your manuscripts at

www.hindawi.com

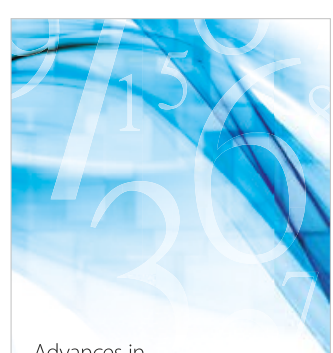

Advances in
Numerical Analysis
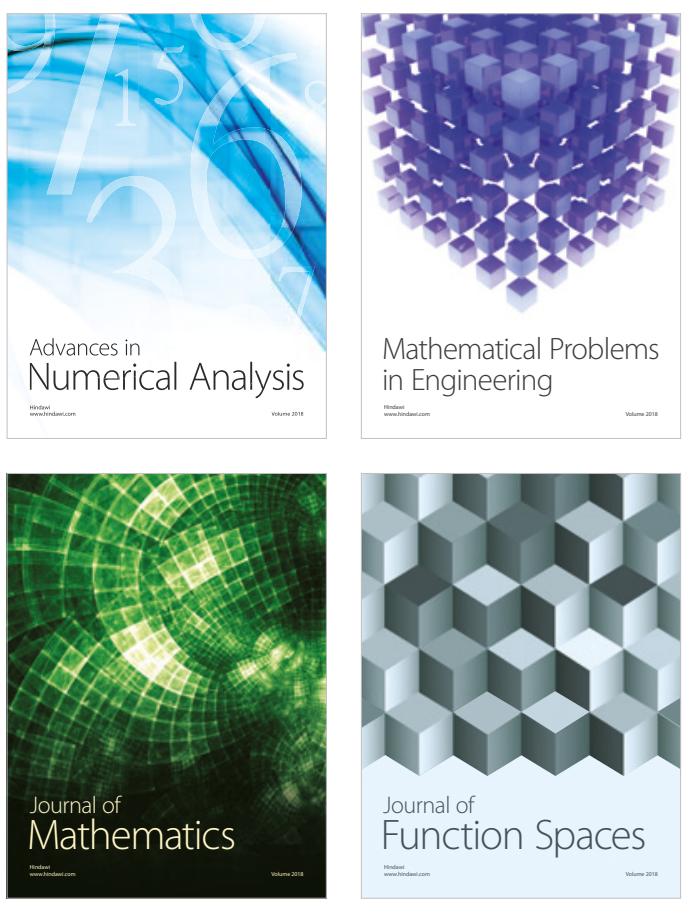

Mathematical Problems in Engineering

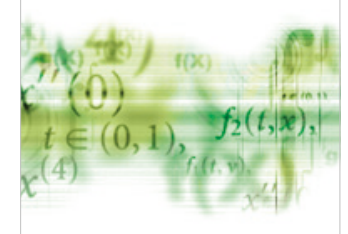

International Journal of

Differential Equations

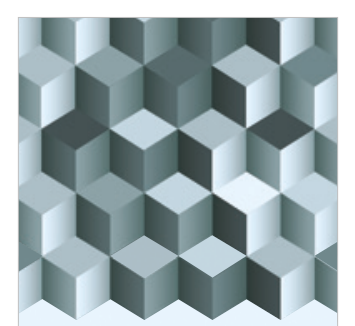

Journal of

Function Spaces

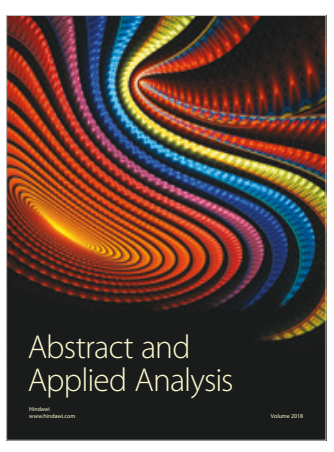

The Scientific

World Journal

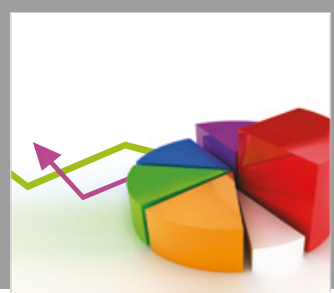

Journal of

Probability and Statistics
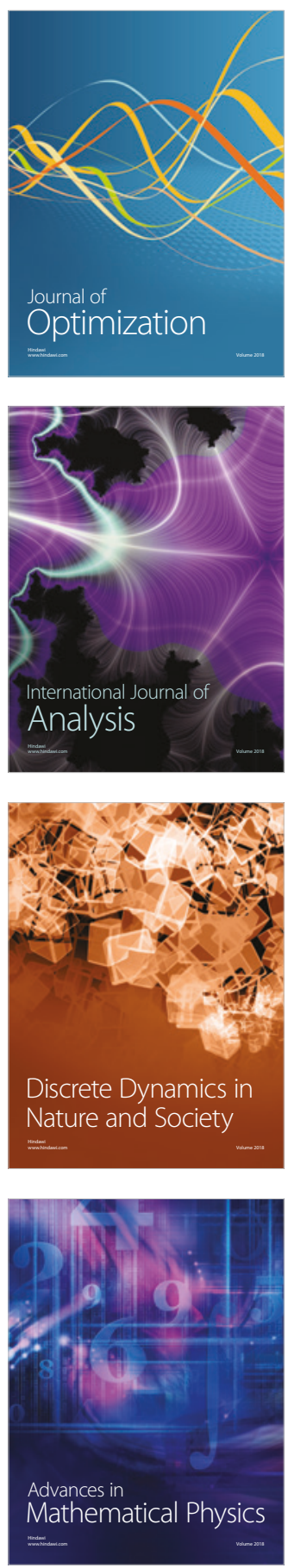